\title{
Phenotypic screening identifies Axl kinase as a negative regulator of an alveolar epithelial cell phenotype
}

\author{
Naoya Fujino ${ }^{1,3,4}$, Hiroshi Kubo ${ }^{2}$ and Rose A Maciewicz ${ }^{1,4}$
}

Loss of epithelial barrier integrity is implicated in a number of human lung diseases. However, the molecular pathways underlying this process are poorly understood. In a phenotypic screen, we identified Axl kinase as a negative regulator of epithelial phenotype and function. Furthermore, suppression of Axl activity by a small molecule kinase inhibitor or downregulation of Axl expression by small interfering RNA led to: (1) the increase in epithelial surfactant protein expression; (2) a cell morphology transition from front-rear polarity to cuboidal shape; (3) the cytoskeletal re-organization resulting in decreased cell mobility; and (4) the acquisition of epithelial junctions. Loss of Axl activity reduced activation of the Axl canonical pathway members, Akt and extracellular signal-regulated kinase-1/2 and resulted in the loss of gene expression of a unique profile of epithelial-to-mesenchymal transition transcription factors including SNAI2, HOXA5, TBX2 or TBX3. Finally, we observed that Axl was activated in hyperplasia of epithelial cells in idiopathic pulmonary fibrosis where epithelial barrier integrity was lost. These results suggest that the Axl kinase signaling pathway is associated with the loss integrity of alveolar epithelium in pathological remodeling of human lung diseases.

Laboratory Investigation (2017) 97, 1047-1062; doi:10.1038/labinvest.2017.52; published online 29 May 2017

Epithelial cells in the respiratory system cover the surface of the airway and secrete a number of anti-microbe molecules including surfactant proteins and act as a front line for innate host defense. ${ }^{1}$ In addition, these cells serve as barriers to prevent entrance of exogenous particles and microbes into lung tissues and the circulation. The barrier integrity depends on tight adhesion of epithelial cells and abundance of fluid and anti-microbial molecules. ${ }^{1}$ The tight adhesion of epithelial cells with adjacent cells is mediated by intercellular adhesion structures including tight junctions and adherens junctions. ${ }^{2}$ Tight junctions are composed of $>40$ proteins, including the zonula occludens-1 (ZO-1), and prevent the flux of fluid between the luminal compartment and the underlying connective tissues. ${ }^{3}$ Although adherens junctions, formed by E-cadherin and $\beta$-catenin, provide a scaffold for the assembly of intracellular actin belts to form stable contacts with neighboring cells. ${ }^{3}$ These adhesion structures are critical for the establishment of apical-basal polarity important for the functional fluid transport and for the secretion of epithelial cells. ${ }^{2}$ Loss of the epithelial barrier integrity is thought to be involved in a number of lung diseases such as idiopathic pulmonary fibrosis (IPF). ${ }^{4,5}$

Human studies and murine experimental models support the concept that the epithelial-to-mesenchymal transition (EMT) program has a key role in loss of the epithelial integrity in pulmonary diseases. ${ }^{6,7}$ Several molecular mechanisms such as mutations of genes encoding surfactant proteins, endoplasmic reticulum stress and the transforming growth factor (TGF) $-\beta$ pathway are proposed to be involved in the EMT followed by the loss of epithelial integrity and pathological remodeling of lung diseases. ${ }^{8}$ However, molecular mechanisms involved in reversing the EMT and restoring the lost epithelial integrity in the lung are not known.

Recent studies suggest the involvement of kinases in controlling the mesenchymal-to-epithelial transition (MET) for generation of induced pluripotent stem cells from mesenchymal fibroblasts. ${ }^{9,10}$ Moreover, multiple kinases such as mitogen-activated protein kinases, receptor tyrosine kinases or phosphatidylinositol kinases have been shown to initiate the EMT. ${ }^{11}$ We hypothesized that kinases have a role

\footnotetext{
${ }^{1}$ Innovative Medicines and Early Development, Respiratory, Inflammation and Autoimmunity, AstraZeneca AB, Mölndal, Sweden and ${ }^{2}$ Department of Advanced Preventive Medicine for Infectious Disease, Tohoku University Graduate School of Medicine, Sendai, Japan

Correspondence: Dr N Fujino, MD, PhD, Department of Respiratory Medicine, Tohoku University Graduate School of Medicine, Sendai 980 8574, Japan or Professor RA Maciewicz, PhD, Innovative Medicines and Early Development, Respiratory, Inflammation and Autoimmunity, AstraZeneca AB, Mölndal SE-431 83, Sweden.

E-mail: nfujino@med.tohoku.ac.jp or Rose.Maciewicz@astrazeneca.com

${ }^{3}$ Current address: Department of Respiratory Medicine, Tohoku University Graduate School of Medicine, Sendai 980 8574, Japan.

${ }^{4}$ These authors equally contributed to this work.

Received 26 January 2017; revised 2 April 2017; accepted 5 April 2017
} 
in regulation of epithelial integrity via the EMT/MET program in pulmonary epithelial cells. Here we screened a diverse set of kinase inhibitors using a phenotypic screen with an untransformed cell line of a multi-potent cell from adult human lung tissues, which had no pathological findings such as tumors, fibrosis or overt inflammation. ${ }^{12}$ This multi-potent cell was unique in that these cells are CD90-expressing mesenchymal stem cell-like cells with low expression of prosurfactant protein-C (proSP-C), which is selectively expressed by alveolar epithelial type II cells (AEC2). ${ }^{13,14}$ These multi-potent cells had high cellular plasticity in vitro as they had the capability of generating $\mathrm{proSP}-\mathrm{C}^{\text {high }} / \mathrm{CD} 90^{\text {neg }}$ AEC2 and proSP-C ${ }^{\text {neg }} / \mathrm{CD} 90^{\text {pos }}$ fibroblasts. ${ }^{12}$

From this study, we identified Axl tyrosine receptor kinase as a negative regulator of an alveolar epithelial phenotype and function through evaluation using an Axl kinase inhibitor and small interfering RNA (siRNA) knockdown approaches. Downstream analysis indicated that Axl pathway canonical members, Akt and extracellular signal-regulated kinase (ERK) $1 / 2$ were involved in this process. We further identified a unique profile of transcription factors controlled under Axl kinase in the MET process. Further evaluation of lung tissues of patients with IPF supported the concept that the Axl kinase was relevant to loss of barrier integrity in the severe lung disease. These results indicate the importance of understanding molecular mechanisms of signal transduction from the outside to the inside of the cell in contributing to the pathological remodeling observed in severe lung diseases.

\section{MATERIALS AND METHODS Isolation and Culture of Human Lung Tissue-Derived Multi-Potent Cells}

Multi-potent cells from adult human lung tissues were isolated as described previously with some modifications. ${ }^{12}$ Lung tissues, which had no any pathological findings such as tumors, fibrosis or overt inflammation, were digested with dispase II (Roche), dispase/collagenase (Roche) and DNase I (Sigma-Aldrich). Single-cell suspensions from which CD45positive cells were removed by autoMACS Separator with anti-human CD45 antibody-coated microbeads (Miltenyi Biotec) were seeded on pre-seeded mouse embryonic fibroblasts (MEFs) that had been treated with MitomycinC (Sigma-Aldrich). Approximately 1 week later, colonies formed by spindle-shaped cells were picked up from each well by pipetting and re-seeded. Sub-confluent cells grown from the re-seeded colonies were trypsinized and passaged. These cell lines were confirmed to be double positive for proSP-C and CD90, and had the ability of secondary colony formation, as well as the capability of in vitro differentiation into AEC2-like cells and mesenchymal cells (osteoblasts and adipocytes). ${ }^{12}$ The presence of the alveolar epithelial marker and the multi-potent differentiation capacity excluded the cell being fibroblasts. Furthermore, contamination of other cell types, such as epithelial cells and endothelial cells, were excluded because of the lack of cell surface markers selective for epithelial cells (EpCAM and E-cadherin) and for endothelial cells (CD31, CD34 and VEGFR2). Cells at passage 5 were used for the phenotypic screen and validation assays.

Lung tissues were provided by patients who underwent lung resection at the Department of Thoracic Surgery at Tohoku University Hospital or the Department of Thoracic Surgery at Japanese Red Cross Ishinomaki Hospital. This study was approved by the Ethics Committee at Tohoku University School of Medicine and Japanese Red Cross Ishinomaki Hospital. Written informed consents were received from all patients before the surgery. The experiments conformed to the principles set out in the WMA Declaration of Helsinki and the Department of Health and Human Services Belmont Report. Information on background of patients from whom multi-potent cells were isolated is shown in Table 1.

\section{Kinase Inhibitor Library}

A kinase inhibitor library covering a broad spectrum of the entire kinome (Supplementary Figure 1) was used for the phenotypic cell-based screen. All of the compounds were dissolved in DMSO $(10 \mathrm{mM})$ and $0.15 \mu \mathrm{l}$ were used per corresponding wells of 96-well polypropylene V-bottom plates (Greiner) by Echo Liquid Handler (LABCYTE). The systematic name of 2D08 is (2S)-1-[4-[4-[6-amino-5-(1,3benzoxazol-2-yl)-3-pyridyl]-3-(methoxymethyl)pyrazol-1-yl]-1piperidyl]-2-hydroxy-propan-1-one (Supplementary Figure 2). The structure of 2D08 was confirmed with NMR and mass spectroscopy using standard techniques.

\section{Phenotypic Screening and Image Acquisition}

Human lung multi-potent cells (Line 1) at passage 5 were plated on 96-well black plates (PerkinElmer, \#6005225) in three replicates at a density of 10000 cells per well in $100 \mu \mathrm{l}$ of growth medium consisting of DMEM, 10\% FBS and penicillin/streptomycin (day 0). At day 1, medium (DMEM, $0.5 \% \mathrm{BSA}$ and penicillin/streptomycin) was put in the compound plates at $150 \mu \mathrm{l}$ per well in order to make $10 \mu \mathrm{M}$ of compound medium. The final concentration of DMSO in this assay for all compounds was $0.1 \%$. Then, the growth medium was replaced with the compound-containing medium. This replacement of medium was repeated every 3 days. Differentiation from human lung multi-potent cells to AEC2 was evaluated by measuring intensity of immunofluorescence staining for proSP-C. At day 13, cells were fixed with $4 \%$ paraformaldehyde, blocked/permeabilized with $10 \%$ goat serum and $0.3 \%$ Triton-X 100 in PBS and stained with antihuman proSP-C antibody (Millipore, \#AB3786, 1:1000) and Alexa Fluor 488-conjugated anti-rabbit antibody (Life Technologies, 1:500). Nuclei were stained with Hochest 33342 (Life Technologies). Concentric 20 images per well were captured by Cellomics ArrayScan (Thermo Scientific) at $\times 20$ magnifications. 
Table 1 Donor information of lung multi-potent cell lines

\begin{tabular}{llllll}
\hline Multi-potent cell & Age & Gender & Indication for surgery & Smoking (pack-years) & Pulmonary function test \\
\hline Line 1 & 16 & Male & Spontaneous pneumothorax & 0 & Not tested \\
Line 2 & 20 & Male & Open lung biopsy (diagnosed as organizing pneumonia) & 6 & Normal \\
Line 3 & 60 & Female & Lung adenocarcinoma & 30 & Normal
\end{tabular}

\section{Image Analysis of the Phenotypic Screen}

The intensity of proSP-C staining and the number of nuclei of the screen were quantified using the Compartmental Analysis algorithm of Cellomics ArrayScan. First, we detected nuclei and positive proSP-C staining as follows: (i) a circle was drawn around a nucleus detected by Hoechst 33342. Dead cells, recognized as bright, aggregated nuclei of Hoechst 33342 staining, were omitted by size and intensity thresholds. (ii) A ring was made to cover most cytoplasm but not the circled nucleus with reference to the nuclear circle. (iii) Positive staining of proSP-C was determined within the ring. Second, we quantified the mean of proSP-C staining intensity per cell as follows. (a) Ring spot total intensity was defined as the sum of the intensity of proSP-C-positive spots by pixel per cell. (b) Ring spot average intensity was defined as ring spot total intensity divided by the number of pixels of proSP-C-positive spots per cell. (c) Mean of ring spot average intensity was defined as the sum of ring spot average intensity by cell divided by the number of cells. In the Result section, we call the mean of ring spot average intensity as mean of proSP-C intensity per cell.

The screen was carried out with three well replicates. If $>90 \%$ of cells were detached, compared with DMSO-treated cell, in two or more wells, the mean of proSP-C intensity of such compounds was recognized as zero. To calculate foldchanges of proSP-C intensity of cells treated with tested compounds compared with DMSO, median of triplicates for each compound was divided by median of DMSO in the same plate. A proSP-C inducer was defined as a small molecule, which showed $>2$-fold increase in proSP-C staining intensity over DMSO.

Immunofluorescence Staining for In Vitro Cultured Cells Human lung multi-potent cells at passage 5 were incubated with DMEM $+0.5 \%$ BSA+penicillin/streptomycin containing either $0.1 \%$ DMSO (vehicle) or a hit compound labeled as 2D08 $(10 \mu \mathrm{M}$; see details in the Result section) on Permanox culture slides (Thermo Scientific) for 6 days. Cells were fixed with $4 \%$ PFA for $30 \mathrm{~min}$ and then blocked and permeabilized with $10 \%$ goat serum (Dako) and $0.3 \%$ Triton-X 100 in PBS for $30 \mathrm{~min}$. Primary antibodies were incubated for $24 \mathrm{~h}$ at $4{ }^{\circ} \mathrm{C}$ as various dilutions as follows rabbit anti-proSP-C antibody (Millipore, \#AB3786, 1:1000), mouse anti-SP-A antibody (Abcam, \#ab51891, 1:1000), rat-anti-T1 $\alpha$ antibody (AngioBio, \#11-009, 1:200), mouse anti-ZO-1 antibody
(Life Technologies, \#33-9100, 1:100), mouse anti-E-cadherin antibody (Life Technologies, \#33-4000, 1:50), rabbit anti- $\beta$ catenin antibody (Cell Signaling Technology, \#8480, 1:100), mouse anti-pan-cytokeratin antibody (Dako, \#M351529, 1:100), mouse anti-vimentin antibody (Dako, \#M072529, 1:100), rabbit anti-SNAI2 antibody (Cell Signaling Technology, \#9585, 1:400), and rabbit anti-Axl antibody (Cell Signaling Technology, \#8661, 1:100). Appropriate secondary antibodies, conjugated with Alexa Fluor 488 or Alexa Fluor 555 (Life Technologies, 1:100), were incubated for $1 \mathrm{~h}$ to detect the primary antibodies. For F-actin staining, Alexa Flour 488-conjugated phalloidin was used according to the instruction (Life Technologies). After mounting of slides and staining of nuclei using ProLong Gold antifade reagent with DAPI (Life Technologies), images were taken via a Nikon C1 confocal microscope (Nikon, Tokyo, Japan). Images were processed using Photoshop CS4 (Adobe).

\section{Dose-Response Curve and Fitting}

Dose-response curves were fitted as previously described. ${ }^{15}$ The compound 2D08 was prepared in 100\% DMSO and serially diluted in DMEM $+0.5 \%$ BSA+penicillin/streptomycin. The final concentration of DMSO for each dilution was $0.1 \%$. The compound was tested in three replicates. GraphPad Prism (GraphPad Software, San Diego, CA, USA) was used to calculate IC50 using the non-linear regression analysis with the $\log$ (inhibitor) $v s$ response - variable slope (four parameters).

\section{Flow Cytometry}

Cell surface antigens were evaluated by flow cytometry with FACSCalibur flow cytometer or BD LSRFortessa (BD Biosciences). Data were analyzed by FlowJo (Tree Star Inc). We used the following antibodies: APC-conjugated mouse anti-CD90 antibody (BD Pharmingen, \#559869, 1:100) and PE-conjugated anti-Axl antibody (R\&D, \#FAB154P dilution, 1:00). For CD90 staining, human lung multi-potent cells were incubated with DMEM+0.5\% BSA+penicillin/streptomycin containing either $0.1 \%$ DMSO or 2D08 $(10 \mu \mathrm{M})$ on Permanox culture slides (Thermo Scientific) for 3 days. For Axl staining, human lung multi-potent cells cultured with DMEM $+10 \%$ FBS+penicillin/streptomycin at passage 5 were analyzed. 


\section{Cell Lysate and Protein Concentration Measurement}

Cells were washed twice with ice-cold PBS and then incubated with ice-cold lysis buffer containing Halt protease/phosphatase inhibitors cocktail (Thermo Scientific) for $5 \mathrm{~min}$ on ice. The lysis buffer consisted of the following: Tris- $\mathrm{HCl}(\mathrm{pH}$ 7.5; $50 \mathrm{mM}$ ), sodium chloride $(\mathrm{NaCl} ; 100 \mathrm{mM})$, ethylenediaminetetraacetic acid (EDTA; $1 \mathrm{mM})$, ethylene glycol tetraacetic acid (EGTA; $1 \mathrm{mM}$ ), Triton-X 100 (1\%), sodium fluoride $(20 \mathrm{mM})$, sodium pyrophosphate $(5 \mathrm{mM})$ and 2 mercaptoethanol $(0.1 \%)$. After incubation, the lysed cells were scraped and collected into $1.5 \mathrm{ml}$ tubes. The lysates were centrifuged at 15000 r.p.m. for $10 \mathrm{~min}$ at $4{ }^{\circ} \mathrm{C}$. Supernatants were aliquoted on ice and stored at $-80^{\circ} \mathrm{C}$. Protein concentration was measured with Pierce $660 \mathrm{~nm}$ Protein Assay (Thermo Scientific) in duplicates. Absorbance at 660 $\mathrm{nm}$ was measured using SpectraMax (Molecular Devices).

\section{Western Blot}

Cell lysates were incubated with NuPAGE LDS Sample Buffer (Life Technologies) and NuPAGE Sample Reducing Agent (Life Technologies) at $98^{\circ} \mathrm{C}$ for $10 \mathrm{~min}$ as per the manufacturer's instruction. After brief centrifugation, lysates were applied to NuPAGE Bis-Tris 4-12\% gel (Life Technologies) for electrophoresis. The proteins were transferred from the gels to nitrocellulose membranes using iBlot Transfer Stack and iBlot Gel Transfer Device (Life Technologies). The membranes were blocked for $2 \mathrm{~h}$ with $5 \%$ non-fat dry milk in Tris-buffered saline with $0.05 \%$ Tween-20 (TBST) and then incubated with the following primary antibodies overnight at $4{ }^{\circ} \mathrm{C}$ : rabbit anti-human Axl antibody (Cell Signaling Technology, \#8661, 1:1000); goat anti-human Gas6 antibody (R\&D, \#AF885, 1:200); rabbit anti-human p-Erk1/2 antibody (Cell Signaling Technology, \#4370, 1:2000); mouse antihuman t-Erk1/2 (Cell Signaling Technology, \#9107, 1:2000); rabbit anti-human p-Akt antibody (Cell Signaling Technology, \#4060, 1:2000); and mouse anti-human t-Akt antibody (Cell Signaling Technology, \#2920, 1:2000). The membrane was further incubated with mouse anti-human $\beta$-actin antibody (Sigma-Aldrich, \#2228, 1:5000) for $30 \mathrm{~min}$ at room temperature and washed with TBST for 5 min three times followed by incubation for $40 \mathrm{~min}$ at room temperature with the following secondary antibodies: IRdye $800 \mathrm{CW}$-conjugated goat anti-rabbit IgG antibody (Rockland, Gilbertsville, PA, USA, \#611-131-003, 1:10000); IRdye 800CW-conjugated goat anti-mouse IgG antibody (Rockland, \#611-132-121, 1:10000); Alexa Fluor 680-conjugated goat anti-mouse IgG antibody (Life Technologies, \#21057, 1:10000); or IRdye $800 \mathrm{CW}$ cojugated donkey anti-goat IgG antibody (LI-COR, Lincoln, NE, USA, \#926-32214, 1:10000). After the washing step, the membranes were scanned with Odyssey Infrared Imaging System (LI-COR).

\section{In Vitro Biochemical Kinase Assay}

We determined IC50 values of the Axl kinase inhibitor (2D08) using kinase-mediated phosphorylation of poly-GAT by AlphaScreen luminescence detection technology. The inhibitor was tested at eight points of dilution in duplicate. Similar methodology was used for screens of all the other kinase inhibitors.

\section{Enzyme-Linked Immunosorbent Assay (ELISA) for Total Axl (t-Axl) and Phosphorylated Axl (p-Axl)}

Cell lysates were normalized for protein concentration. t-Axl and p-Axl were measured by PathScan Total Axl Sandwich ELISA kit and PathScan Phospho-Axl (panTyr) Sandwich ELISA kit according to the manufacturer's instruction (Cell Signaling Technology). To determine the ratio of p-Axl to $\mathrm{t}$-Axl for the dose-response experiment, backgroundcorrected absorbance at $450 \mathrm{~nm}$ of $\mathrm{p}$-Axl was divided by that of t-Axl. To quantify relative amount of $\mathrm{p}$-Axl in the siRNA knockdown study, a standard curve was generated using pooled lysates of cells transfected with non-targeted siRNA.

\section{Kinome Mapping}

We utilized the Kinome Render (http://bcb.med.usherbrooke. $\mathrm{ca} /$ kinomerender.php) to make an annotated kinome tree indicating interaction between the kinase inhibitor library and kinases based on binding data of the in vitro biochemical assays. ${ }^{16}$

\section{siRNA-Mediated Knockdown}

Knockdown of Axl was conducted by specific single siRNAs using Silencer Select siRNAs for Axl (Life Technologies, Ambion \#s1845 (siAxl-a), \#s1846 (siAxl-b) and \#s1847 $($ siAxl-c)). Silencer Select Negative control \#1 siRNA (Life Technologies) was used as non-targeted siRNA. Lipofectamine RNAiMAX (Life Technologies) was used to introduce siRNAs to multi-potent cells. Mock transfections contained lipofectamine solution without siRNA. Untreated samples did not contain siRNAs and lipofectamine. Transient transfection was performed according to the manufacturer's instruction. Knockdown efficacy at different times or with different concentrations was evaluated by western blot and ELISA. We determined the working concentrations of siRNAs and lipofectamine as $10 \mathrm{nM}$ and $0.1 \%$, respectively. For the evaluation of knockdown efficacy, multi-potent cells were seeded at a density of 85000 cells per well on a 12-well format plate in DMEM+10\% FBS. For inducing differentiation, cells were seeded at a density of 3000 cells per well on a 96-well format plate (PerkinElmer, \#6005225) in the same medium. After $2 \mathrm{~h}$, the medium was replaced with DMEM $+0.5 \%$ BSA followed by transfecting the multi-potent cells with siRNAlipofectamine complexes. Knockdown efficacy and differentiation capability were evaluated 3 days after the transfection.

\section{Image Analysis of Immunostaining of proSP-C and SNAI2 in siRNA-Mediated Knockdown Experiments}

Concentric 25 images per well were captured by Cellomics ArrayScan (Thermo Scientific) at $\times 20$ magnifications. Here proSP-C-positive cells were defined as cells showing a 
granular pattern of proSP-C staining in cytoplasm. For quantification of SNAI2 staining, a circle was drawn around a nucleus detected by Hoechst 33342. We measured the intensity of SNAI2 staining in the nucleus. Dead cells, recognized as bright aggregated nuclei of Hoechst 33342 staining, were omitted by size and intensity thresholds.

\section{Migration Assay}

Human lung multi-potent cells at passage 5 were plated at a density of 250000 cells per well in six-well plates (Corning) with growth medium (DMEM containing 10\% FBS and penicillin/streptomycin). After $24 \mathrm{~h}$, multi-potent cells were incubated with $\mathrm{DMEM}+0.5 \% \mathrm{BSA}+$ penicillin/streptomycin containing $0.1 \%$ DMSO (vehicle) or 2D08 $(0.1,1,10 \mu \mathrm{M})$ for $3 \mathrm{~h}$ in a humidified $5 \% \mathrm{CO}_{2}$ incubator at $37^{\circ} \mathrm{C}$. These cells were trypsinized and seeded at 20000 cells per well in three replicates on 12 -well cell culture transwell inserts with $8 \mu \mathrm{m}$ pore size (Transwell Permeable Supports, Corning) with DMEM+0.5\% BSA+penicillin/streptomycin. Lower transwell chambers contained DMEM+10\% FBS+penicillin/streptomycin were used to allow cells to migrate. $0.1 \%$ DMSO or 2D08 was added to corresponding upper and lower transwell chambers. After $16 \mathrm{~h}$, non-migrated cells were removed by cotton swabs. Migrated cells were fixed with $4 \%$ PFA, permeabilized with methanol and stained with crystal violet (Sigma-Aldrich). The field-images per transwell were taken by an inverted light microscope (Eclipse TE2000, Nikon). Data are presented as the number of migrated cells per $\mathrm{mm}^{2}$.

\section{RNA Extraction and RT-qPCR}

For examination of mRNA expression of SFTPC, THY1 (CD90), SNAI1, SNAI2, ZEB1, ZEB2, and 12 transcription factors identified through Affimetrix microarray analysis (HOXA5, TBX2, TBX3, NR2F1, FOXF1, HOXA3, ZFPM2, FOXF2, TWIST1, FOXD1, HOXB3 and HOXB7), human lung multi-potent cells at passage 5 were incubated with DMEM $+1 \%$ FBS+penicillin/streptomycin containing either $0.1 \%$ DMSO (vehicle) or 2D08 $(10 \mu \mathrm{M})$ on six-well culture plates (Corning) for forty-eight hours (SFTPC, THY1, SNAI1, $S N A I 2, Z E B 1$ and $Z E B 2$ ) or $4 \mathrm{~h}$ (the 12 transcription factors). Total RNA was extracted using RNeasy Micro Kit Plus (Qiagen). The total RNA was assessed for quantity and quality using UV spectrophotometry using a ND-100 spectrophotometer (Nano Drop Technologies, Rockland, DE, USA). cDNA was synthesized from the total RNA ( $1 \mu \mathrm{g}$ for each sample) using QuantiTect Reverse Transcription Kit according to the manufacturer's instruction (Qiagen). Quantitative real-time PCR was conducted using TaqMan assays (Life Technologies) on Applied Biosystems 7500 Real-Time PCR System (Life Technologies) in duplicates. We used the following program: UDG incubation at $50{ }^{\circ} \mathrm{C}$ for $2 \mathrm{~min}$, AmpliTaqGold activation at $95^{\circ} \mathrm{C}$ for $10 \mathrm{~min}, 45$ cycles of amplification repeats (denature at $95^{\circ} \mathrm{C}$ for $15 \mathrm{~s}$ and anneal/ extension at $60^{\circ} \mathrm{C}$ for $1 \mathrm{~min}$ ). Seven housekeeping genes (GAPDH, RPLPO, TBP, $18 \mathrm{~S}$ rRNA, HPRT, PGK and ACTB) were tested to select an endogenous control, which minimized deviation of raw $\mathrm{Ct}$ values under several culture conditions using the same quantity of templates. RPLPO was selected as the best endogenous control for this assay (s.d. of raw Ct: RPLPO, 0.31; 18S rRNA, 0.32; GAPDH, 0.50; TBP, 0.50; $P G K, \quad 0.50 ;$ HPRT, 076; ACTB, 1.32). We used the following TaqMan assays: SFTPC, Hs00161628_m1; THY1, Hs00264235_s1; ZEB1, Hs00232783_m1; SNAI1, Hs0 0195591_m1; SNAI2, Hs00950344_m1; NR2F1, Hs00818 842_m1; FOXF2, Hs00230963_m1; FOXF1, Hs002309 62_m1; TBX3, Hs00195612_m1; HOXB3, Hs01587922_m1; ZEB2, Hs00207691_m1; TBX2, Hs00911929_m1; HOXA5, Hs00430330_m1; FOXD1, Hs00270117_s1; TWIST1, Hs016 75818_s1; ZFPM2, Hs00201397_m1; HOXA3, Hs006 01076_m1; HOXB7, Hs00270131_m1; RPLP0, Hs99999902_m1. Relative quantification of mRNA expression was performed with standard curves generated from total RNA of the undifferentiated human lung multi-potent cell line or a whole lung tissue homogenate (Takara Bio Europe/ Clontech, Saint-Germain-en-Laye, France). The quantified transcripts were then normalized by RPLPO. The normalized mRNA amounts in cells treated with 2D08 were compared with those in DMSO-treated cells.

\section{Microarray Analysis}

Affymetrix gene expression array data of human lung multipotent cell lines (GEO Series accession no. GSE21095) were downloaded from the NCBI Gene Expression Omnibus (http://www.ncbi.nlm.nih.gov/geo). Statistics for gene expression levels were computed using Array Studio (Omicsoft, Cary, NC, USA). To perform a probe sequence analysis of present/absent call, we normalized the data with the Microarray Suite version 5.0 (MAS 5.0) algorithm. Genes flagged as present call in each of the three cell lines were considered to be expressed in human lung multi-potent cells.

\section{Immunohistochemistry for Human Lung Specimens}

Lung tissues were fixed with $10 \%$ formalin, embedded in paraffin and sectioned at a $3 \mu \mathrm{m}$ thickness. Immunohistochemistry for Axl and Gas6 was performed using the Envision polymer method (Histofine MAX-PO kit, Nichirei, Tokyo, Japan). Heat-induced antigen retrieval by autoclave was performed using basic retrieval solution (R\&D, cat\# CTS013) for $5 \mathrm{~min}$ at $121^{\circ} \mathrm{C}$ for the Axl and Gas6 staining. The sections were blocked with $10 \%$ rabbit serum for Axl and Gas6 staining and were then incubated at $4{ }^{\circ} \mathrm{C}$ overnight with the following primary antibodies: goat anti-human Axl antibody $(\mathrm{R} \& \mathrm{D}$, cat\# AF154, 1:100); goat anti-human Gas6 antibody (R\&D, cat\# AF885, 1:20). Endogenous peroxidase activity was blocked with $0.3 \% \mathrm{H}_{2} \mathrm{O}_{2}$ in methanol. The sections were subsequently reacted with a sufficient quantity of secondary antibodies conjugated with polymer and peroxidase (Nichirei). The immune complex was visualized with a 3,3'-diaminobenzidine (DAB) solution. Hematoxylin was used for counterstaining the nuclei. Nonreactive immunoglobulin of the same isotype was 

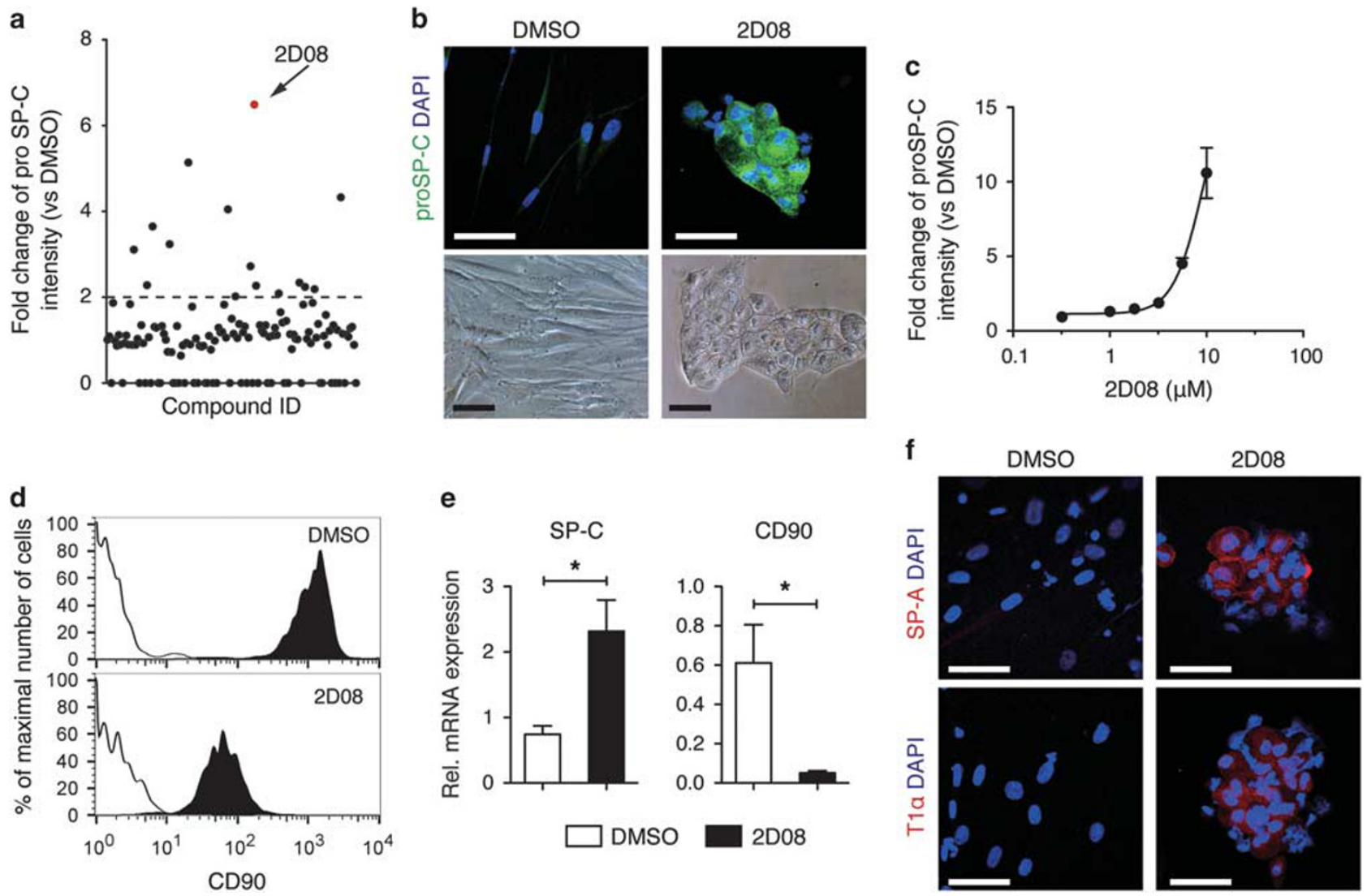

Figure 1 A phenotypic screen identifies a kinase inhibitor to induce alveolar epithelial phenotype of human lung multi-potent cells in vitro. (a) Screening of the compound library for their ability to induce proSP-C. Data are shown as the fold change in proSP-C intensity of each compound compared with $0.1 \%$ DMSO. Cut-off value shown as a dotted line was defined as twofold. (b) Immunofluorescence images of proSP-C (green) and morphologic appearance of human lung multi-potent cells treated with DMSO (left panels) or the hit compound labeled as 2D08 (right panels). (c) The dose-dependent effect of the Axl kinase inhibitor on proSP-C intensity. The $x$ axis represents a log scale. (d) Flow cytometric analysis showing downregulation of the lung multi-potent cell marker CD90 by 2D08. (e) qRT-PCR analysis of changes in SP-C and CD90 mRNA expression by 2D08. (f) Immunostaining analysis for another AEC2 marker (surfactant protein-A (SP-A; top, red)) and for an alveolar epithelial type I cell marker, T1a (bottom, red) of human lung multi-potent cells treated with DMSO (left panels) or 2D08 (right panels). Data are mean \pm s.e.m. of three individuals (c, e). ${ }^{*} P<0.05$ vs DMSO; two-tailed unpaired $t$-test (e). Nuclei (blue) were visualized with DAPI (b, f). Scale bars represent $50 \mu \mathrm{m}(\mathbf{b}, \mathbf{f})$.

used as a negative control. Images were captured using a Nikon Eclipse microscope (Nikon). Information on background of patients for the histological analysis is shown in Table 2.

\section{Immunofluorescence Staining for Human Lung Specimens}

Lung tissues were fixed with $10 \%$ formalin, embedded in paraffin and sectioned at a $3 \mu \mathrm{m}$ thickness. Double immunofluorescence staining of $\mathrm{Axl}$ in combination with pancytokeratin, proSP-C, CD90 or CD31 was performed. Heatinduced antigen retrieval by autoclave (for $5 \mathrm{~min}$ at $121{ }^{\circ} \mathrm{C}$ ) was performed using basic retrieval solution $(\mathrm{R} \& \mathrm{D}$, cat\# CTS013) for the staining of Axl with pan-cytokeratin, proSP$\mathrm{C}$ or $\mathrm{CD} 31$ or using antigen retrieval solution at $\mathrm{pH} 9$ (Nichirei) for the staining of Axl and CD90. Sections were blocked and permeabilized for $30 \mathrm{~min}$ at room temperature with $0.3 \%$ Triton-X100/10\% donkey serum/PBS. The sections were incubated overnight at $4{ }^{\circ} \mathrm{C}$ with primary antibodies as follows: goat anti-human Axl antibody (R\&D, cat\# AF154, 1:100); mouse anti-pan-cytokeratin antibody (Dako, cat\# M351529, 1:100); rabbit anti-proSP-C antibody (Millipore, cat\# AB3786, 1:1000); mouse anti-CD90 antibody (Serotec, cat\# MCA90, 1:50); mouse anti-CD31 antibody (Dako, cat\# M0823, 1:40). Secondary antibodies were incubated using Alexa Fluor 546-conjugated donkey anti-goat antibody, Alexa Fluor 488-conjugated donkey anti-rabbit antibody or Alexa Fluor 488-conjugated donkey anti-mouse antibody (Life Technologies, 1:100). After mounting of slides and staining of nuclei using ProLong Gold antifade reagent with DAPI (Life Technologies), images were taken via a Nikon C2 confocal microscope (Nikon). Projection images were shown. The images were processed using Photoshop CS4 (Adobe).

\section{Statistics}

Statistical analyses were conducted with GraphPad Prism Version 5.0d (GraphPad Software). Data were compared 
Table 2 Patients' information on the immunohistochemical analysis

\begin{tabular}{llllc}
\hline & Age & Gender & $\begin{array}{l}\text { Indication for } \\
\text { surgery }\end{array}$ & $\begin{array}{c}\text { Smoking history } \\
\text { (pack-years) }\end{array}$ \\
\hline Normal 1 & 62 & Male & Lung cancer & 70 \\
Normal 2 & 56 & Male & Lung cancer & 2 \\
Normal 3 & 69 & Male & Lung cancer & 40 \\
IPF 1 & 80 & Male & Lung cancer & 0 \\
IPF 2 & 82 & Male & Biopsy & 0 \\
IPF 3 & 69 & Male & Lung cancer & 40 \\
\hline
\end{tabular}

Abbreviation: IPF, idiopathic pulmonary fibrosis.

Normal indicates tissues without any overt inflammation, fibrosis and cancer.

using a two-tailed unpaired $t$-test or a one-way analysis of variance (ANOVA). When the overall differences were identified in ANOVA, a Bonferroni's multiple comparison test was performed to identify which groups were significantly different. The percentage of migrated cells between different concentrations of the hit compound was compared using Kruskal-Wallis test with Dunn's multiple comparison test. The relative amount of mRNA of SNAI1, SNAI2, ZEB1 and ZEB2 in three lines of human lung multi-potent cells was compared using paired $t$-test. Statistical significance was defined as $P<0.05$.

\section{RESULTS}

Identification of a Kinase Inhibitor that Induces Alveolar Epithelial Cell Phenotypes in Human Lung Multi-Potent Cells

A cell-based phenotypic screen using a diverse kinase inhibitor collection was undertaken to assess molecular mechanisms involved in induction of proSP-C as a hallmark of alveolar epithelial type II cell (AEC2) function. ProSP-C was used as an increase in its expression indicative of differentiation to AEC2 from pluripotent stem cells or other lung stem/progenitor cell populations. ${ }^{17-19}$ Through the screen, we identified 15 compounds that induced proSP-C by $>2$-fold staining over DMSO (Figure 1a,Supplementary Figure 3 and Supplementary Table 1). Analysis of the effect on the morphology, as a secondary measure of acquisition of epithelial cell integrity, indicated that only 1 of these 15 proSP-C inducers, a compound labeled as 2D08, could promote a distinct morphological change (Figure 1b). Here treatment reduced front-rear polarity, a morphological trait of mesenchymal cells and induced cuboidal cell morphology, a feature of an alveolar epithelial phenotype. The remaining 14 proSP-C inducers did not clearly induce this epithelial morphology (Supplementary Figure 3 and Supplementary Table 1). This screening result was validated on three individual lines of human lung tissue-derived multi-potent cells, in a dose-response analysis of the compound (Figure 1c). In accordance with our previous report showing that expression of CD90 (a marker of mesenchymal stem cells) was decreased during epithelial differentiation, ${ }^{12}$ 2D08 treatment diminished CD90 protein expression (Figure 1d). The increase of SP-C and decrease of CD90 was further verified with qRT-PCR analysis (Figure 1e). Here, mRNA of SP-C or CD90 was found to be upregulated $(3.30 \pm 0.91$-fold, mean \pm s.e.m. of three cell lines; $P=0.033$ ) or downregulated $(0.10 \pm 0.02$ fold, mean \pm s.e.m. of three cell lines; $P=0.034)$, respectively. To further validate the effect of compound 2D08 on the acquisition of alveolar epithelial phenotypes, we explored expression of other markers. Immunostaining confirmed that another AEC2 marker (surfactant protein-A, SP-A) and an alveolar epithelial type I cell (AEC1) marker (T1 $\alpha$, also known as podoplanin) were upregulated in the cells after treatment with 2D08 (Figure 1f).

\section{Suppression of Axl Expression Induces ProSP-C in Human Lung Multi-Potent Cells}

Evaluation of the kinase inhibition profile of 2D08 by in vitro biochemical assays of kinases indicated that 2D08 inhibited nine kinases with IC50 values $<100 \mathrm{nM}$ (Supplementary Table 1). The strongest potency was against Axl (IC50 $=0.49 \mathrm{nM}$ ). Furthermore, of those kinases that had potency 100 -fold or less than that against Axl, only four (interleukin-1 receptorassociated kinase 4 (IRAK4), glycogen synthase kinase-3 $\beta$ (GSK-3 $\beta$ ), phosphatidylinositol-4,5-bisphosphate 3-kinase $\alpha$ $(\mathrm{PI} 3 \mathrm{~K} \alpha)$ and epidermal growth factor receptor (EGFR)) were expressed by the representative lung multi-potent cell lines (Supplementary Table 2). Of these, we found that selective GSK-3 $\beta$ inhibitors (CHIR99021 and Kenpaullone, labeled as $1 \mathrm{C} 03$ and $2 \mathrm{H} 06$, respectively) and a PI3K inhibitor (NVPBEZ235 (2D05)) failed to induce the epithelial morphology in the phenotypic screen (Supplementary Figure 3 and Supplementary Table 1). Furthermore, other PI3K inhibitors (LY294002 (1C06) and Wortmannin (1C02)) evaluated did not increase proSP-C expression >2-fold of DMSO (LY294002, 1.03-fold change; Wortmannin, 0.89 fold change). Finally, we observed that the kinase inhibitors 1B05 (IC50 for IRAK4 $=31 \mathrm{nM}$ ) and 2B02 (IC50 for EGFR $=2.2 \mathrm{nM}$ ) also did not induce the epithelial shape (data not shown).

Based on these results, we focused on the role of Axl in the transition to alveolar epithelial phenotype. The expression of Axl kinase in our human lung multi-potent cells was verified by western blot analysis, detecting bands at 140 and $120 \mathrm{kDa}$ (Figure 2a), which corresponded to the fully and partially glycosylated Axl..$^{20}$ Growth arrest-specific-6 (Gas6), a ligand for Axl, was also expressed (Figure 2a). Immunostaining of permeabilized cells using an antibody recognizing a cytoplasmic domain of Axl showed a cytoplasmic pattern of Axl staining with greater staining around the edge of the cell (Figure 2b). A flow cytometric analysis confirmed that Axl was expressed on the surface of the lung multi-potent cells (Figure 2c). Furthermore, the phosphorylation status was confirmed by ELISA for detection of total phosphorylated tyrosine on Axl. Evaluation of the ratio of p-Axl to t-Axl 
a

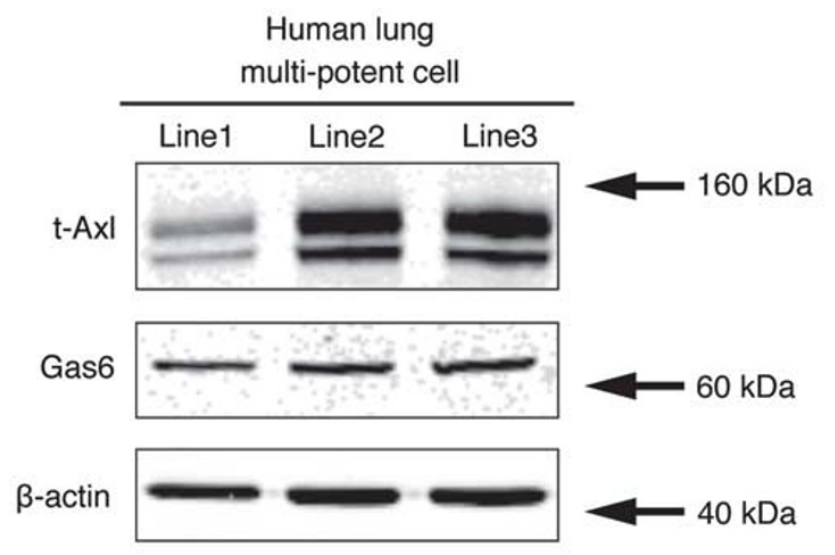

b
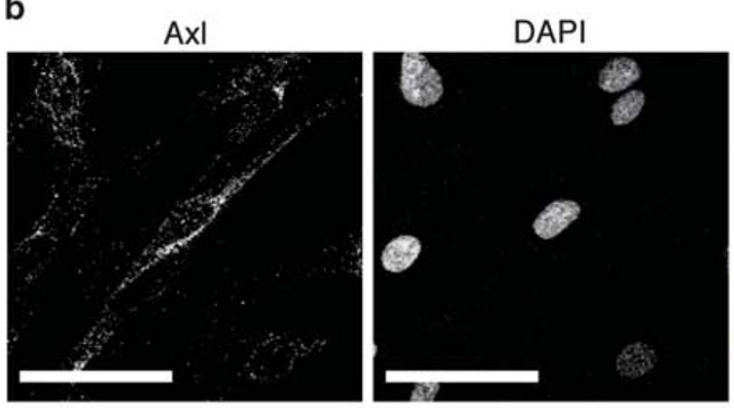

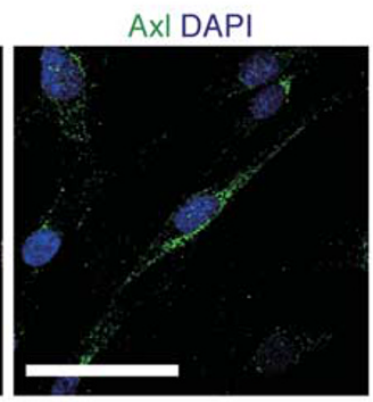

C
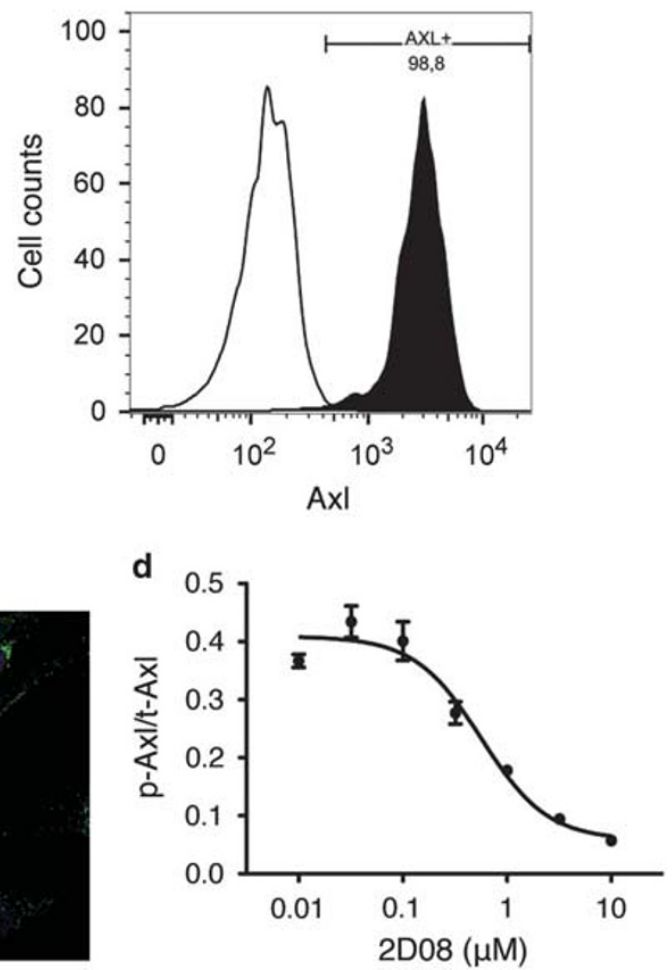

Figure 2 TAM receptor tyrosine kinase Axl is targeted by the hit compound (labeled as 2D08) in human lung multi-potent cells. (a) Protein expression of Axl kinase and Gas6 in the three multi-potent cell lines under an undifferentiated condition. $\beta$-Actin served as an internal control. (b) Representative immunostaining for Axl indicating membranous and perinuclear staining patterns. Nuclei were stained with DAPI. Scale bars represent $50 \mu \mathrm{m}$. (c) Flow cytometric analysis indicates cell surface expression of Axl. Specific staining, shaded. Isotype control, open. (d) A dose-dependent decrease in the ratio of p-Axl to t-Axl by 2D08. Data are mean \pm s.e.m. of three individuals. The $x$ axis represents concentration of the hit compound 2D08 in a log scale.

indicated that 2D08 decreased the ratio of p-Axl to t-Axl in a dose-dependent manner (Figure 2d), indicating that this Axl expression was functional.

To determine that Axl was a negative regulator kinase in the observed alveolar epithelial transition of human lung multi-potent cells, Axl was selectively knocked down using three unique siRNAs (called here siAxl-a, siAxl-b and siAxl-c). Western blot analysis showed high knockdown efficacy (Figure 3a) and ELISA analysis demonstrated that the amount of p-Axl was also decreased (Figure 3b) by all three siRNAs. The percent inhibition of p-Axl was $93.8 \pm 2.0$ (siAxl-a), $89.3 \pm 1.8$ (siAxl-b), $94.7 \pm 1.5$ (siAxl-c) compared with non-targeted siRNA (mean \pm s.e.m. of three multipotent cell lines). We found that knockdown of Axl significantly increased the number of proSP-C-expressing cells compared with non-targeted siRNA (Figures $3 \mathrm{c}$ and d). Silencing of Axl also enhanced the epithelial shape in the human lung multi-potent cells with the concomitant loss of spindle shape and the front-rear polarity (Figure 3e).

\section{Inhibition of Axl Activity Promotes Epithelial Integrity in Human Lung Multi-Potent Cells via MET}

We evaluated the role of Axl kinase inhibition on epithelial junction and cytoskeleton organization during the transition from the mesenchymal morphology to the epithelial shape. We observed that suppression of Axl kinase activity by 2D08 (hereafter called Axl inhibitor) disrupted the cytoskeleton and actin filaments with re-organization at cellular junctions as shown by F-actin staining with phalloidin (Figure 4a) and increased expression of ZO-1 (a tight junction protein) at cellular junctions (Figure 4a). The Axl inhibitor also promoted translocation of $\beta$-catenin to cellular junctions (Figure 4b) and caused an upregulated E-cadherin (Figure 4c), both of which are components at adherens junction. Furthermore, inhibition of Axl kinase activity increased expression of cytokeratin, a component of epithelial intermediate filaments (Figure $4 \mathrm{~d}$ ) and decreased vimentin expression, a component of mesenchymal intermediate filaments (Figure 4e). siRNA-targeted Axl knockdown also increased expression of ZO-1 and promoted translocation of $\beta$-catenin to cellular junctions (data not shown).

Motility is a cellular function of mesenchymal cells and gain of motility is associated with cytoskeletal re-organization during EMT. ${ }^{21}$ We investigated the migration ability of human lung multi-potent cells treated with different concentration of the Axl kinase inhibitor. Inhibition of Axl kinase activity significantly decreased the migration ability in all three cell lines in a dose-dependent manner (Figure 4f). 
a

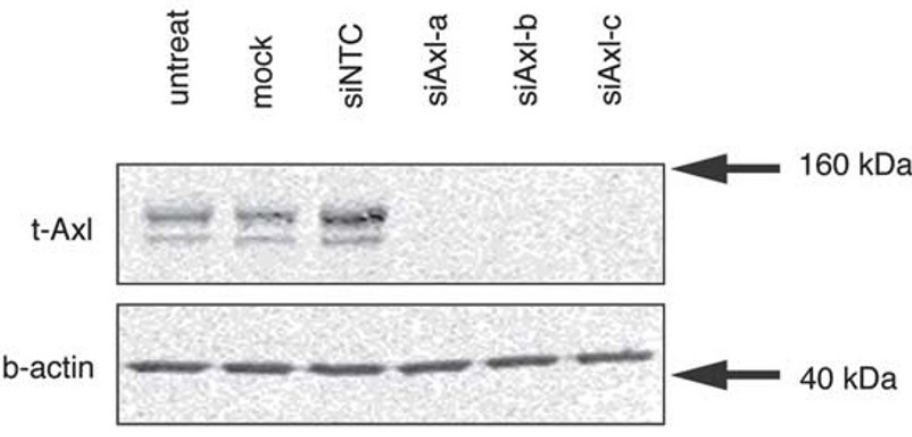

C
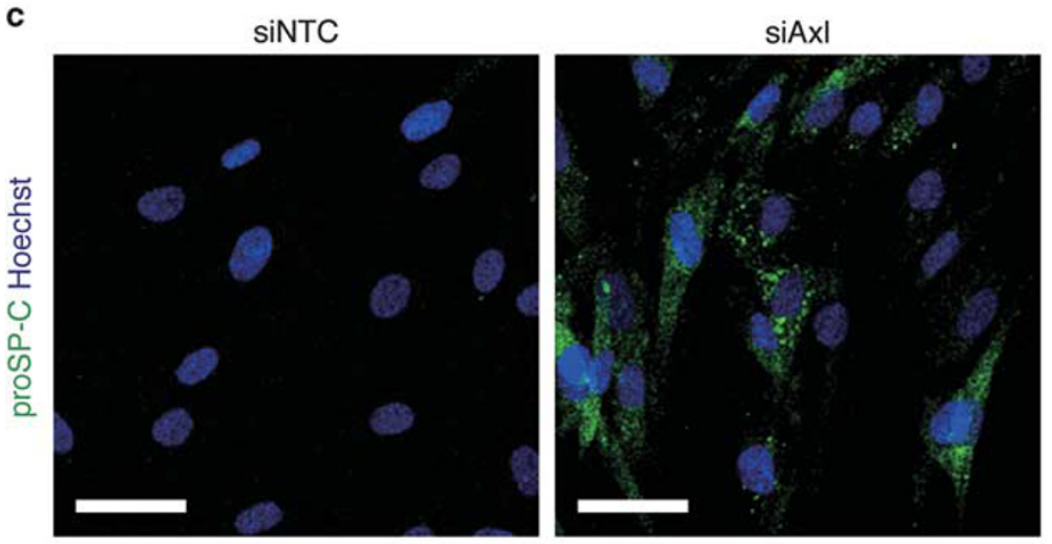

e

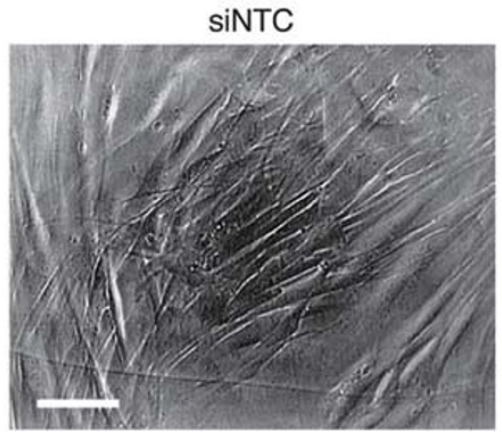

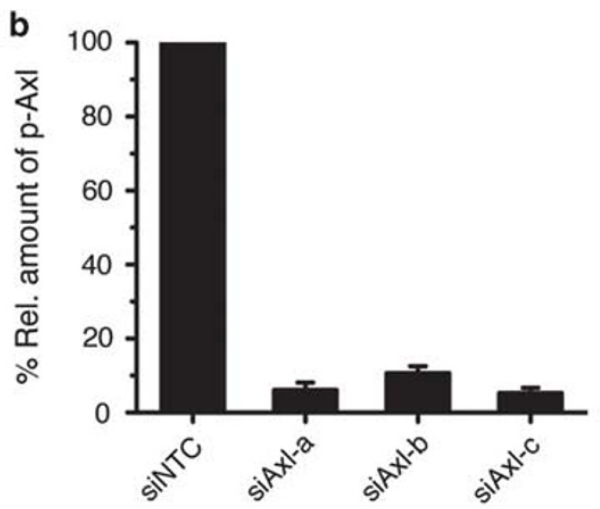

d

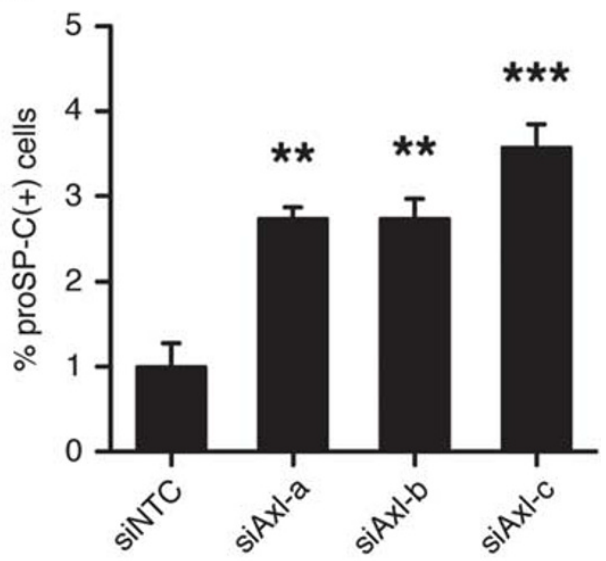

Figure 3 Knockdown of Axl induces transition of human lung multi-potent cells to AEC2 phenotypes. (a) Effect of treatment with three individual siRNAs targeting $\mathrm{Axl}(\mathrm{siAxl})$ on expression of $\mathrm{Axl}$ protein in a human lung multi-potent cell line (Line 1). Untreated cells (untreat), cells treated with only lipofectamine (mock) and cells treated with non-targeted siRNA with lipofectamine (siNTC) were used for transfection controls. $\beta$-Actin is used as an internal control in immunoblot. (b) ELISA analysis for relative quantification of p-Axl after transfection of Axl-targeted siRNAs in human lung multi-potent cell lines. (c) Representative immunostaining for proSP-C (green) in the presence of non-targeted siRNA (left) or Axl-targeted siRNA (right). Nuclei (blue) were stained with Hoechst 33342. (d) Effect of treatment (as described in a) on the number of proSP-C-expressing cells. (e) Phase contrast images of human lung multi-potent cells (Line 1) after treatment with non-targeted siRNA (left) or Axl-targeted siRNA (right). Data are mean \pm s.e.m. of three individuals (b, d). ${ }^{* *} P<0.01,{ }^{* *} P<0.001$ vs siNTC; one-way analysis of variance with Bonferroni's multiple comparison test (d). Scale bars represent $50 \mu \mathrm{m}(\mathbf{c}, \mathbf{e})$.

\section{Inhibition of AxI Activity Decreased Phosphorylation of AKT and ERK1/2, and Downregulated Transcription Factors SNAI2, HOXA5 and TBX2/3}

To understand how Axl may transduce its effect in promoting the epithelial transition, we explored the effect of inhibition or activation as assessed by phosphorylation of members of its canonical pathway. ${ }^{22}$ We cultured the human lung multipotent cells with the Axl inhibitor for $3 \mathrm{~h}$ and found that inhibition of Axl activity lowered the phosphorylation of both Akt and ERK1/2 in a dose-dependent manner although the dose required appear to be less for inhibition of Akt than ERK1/2 (Figure 5). 
a
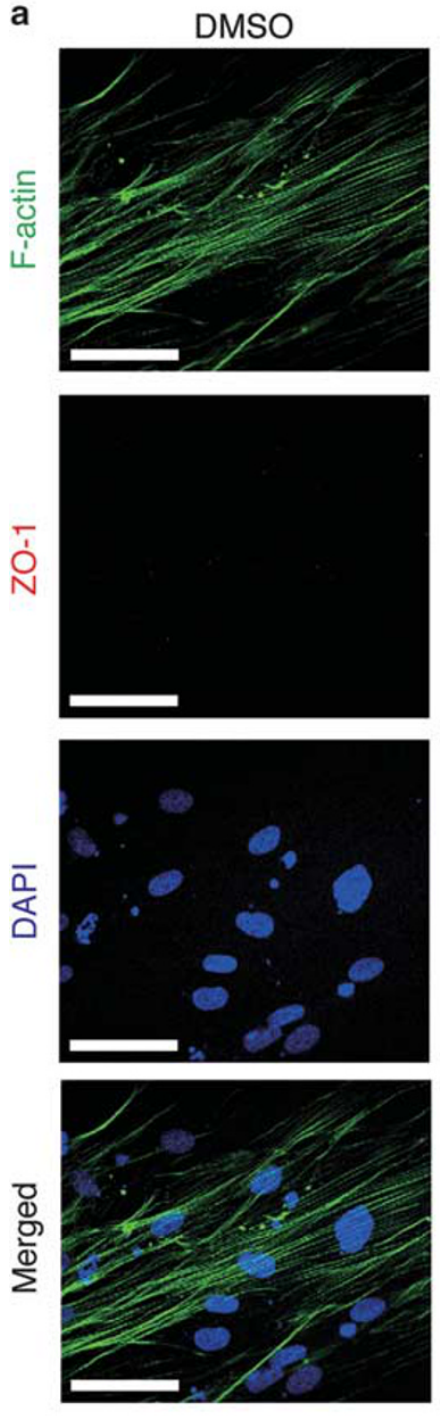

f

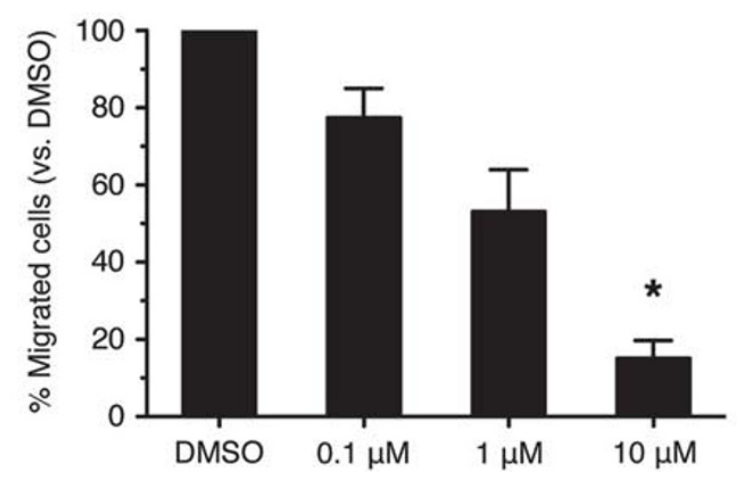

b
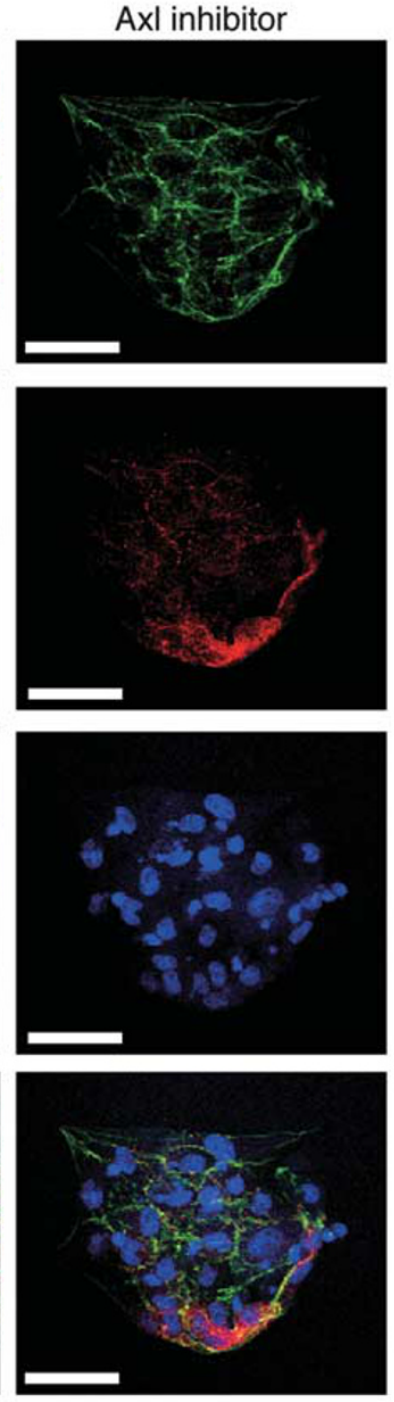

d
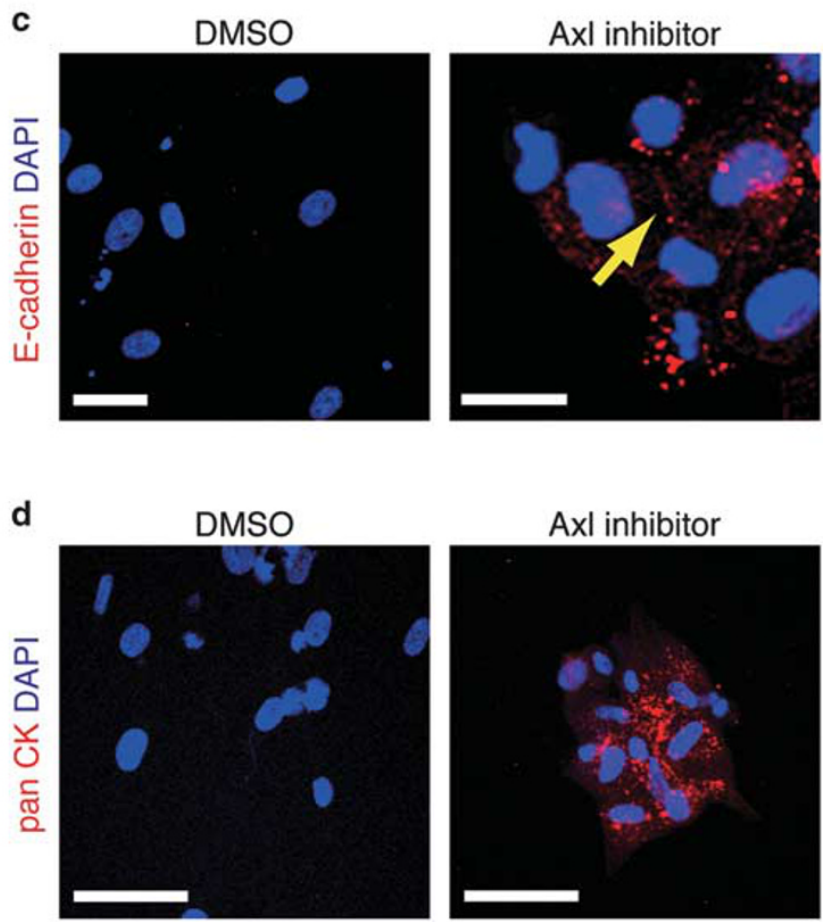

e

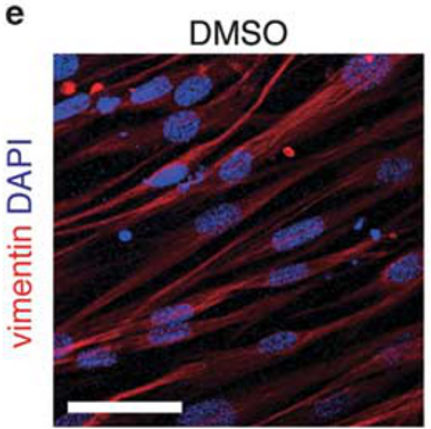

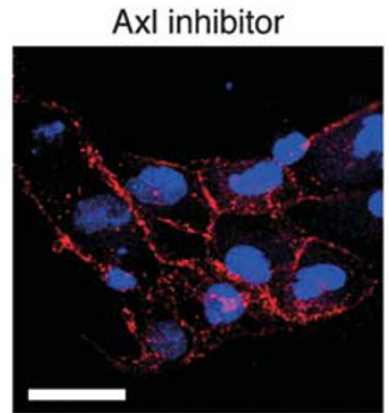

Axl inhibitor

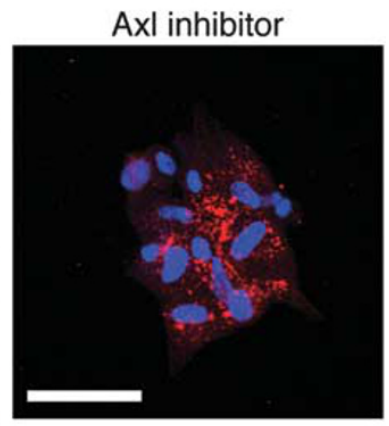

Axl inhibitor

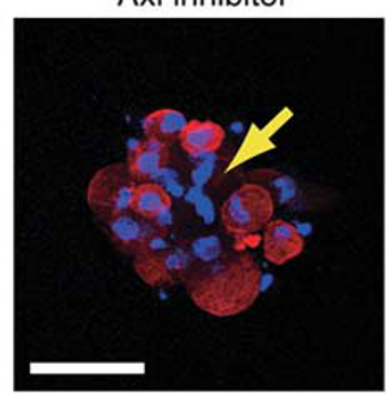

Figure 4 Inhibition of Axl kinase forms epithelial junctions in human lung multi-potent cells via MET. Visualization of epithelial cell junctions and cytoskeleton in the presence or absence of the Axl kinase inhibitor (2D08) by F-actin staining (a, green) or by immunostaining for ZO-1 (a, red), $\beta$-catenin (b, red), e-cadherin (c, red; an arrow indicates staining at cellular junction), pan-cytokeratin (pan CK; $\mathbf{d}$, red) and vimentin (e, red; an arrow indicates vimentin-negative cells). Nuclei are stained with DAPI (a-e). Scale bars represent $50 \mu \mathrm{m}$ (a, b, d, e) and $25 \mu \mathrm{m}$ (c). (f) Effect of the Axl inhibitor 2D08 on the migration ability. Data are mean \pm s.e.m. of three individuals. ${ }^{*} P<0.05$ vs DMSO; Kruskal-Wallis test with Dunn's multiple comparison test. 


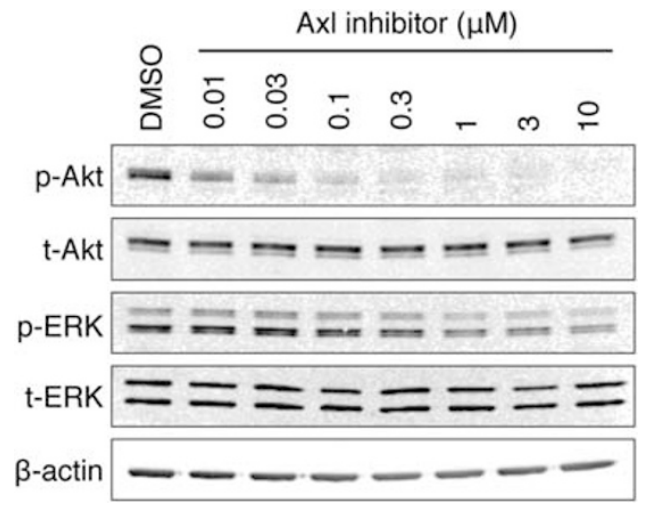

Figure 5 Downregulation of canonical downstream kinase pathways of Axl by the Axl inhibitor 2D08. Immunoblots for phosphorylated Akt (p-Akt), total-Akt (t-Akt), phosphorylated ERK1/2 (p-ERK1/2) and total ERK1/2 (t-ERK1/2) in the presence of DMSO or Axl inhibitor at seven point concentrations. $\beta$-Actin served as an internal control.

We also explored the downstream effect of inhibition of Axl kinase on the expression of transcription factors. We initially focused on snail family zinc-finger 1 and 2 (SNAI1 and SNAI2) and zinc-finger E-box binding homeobox 1 and 2 (ZEB1 and ZEB2). These transcription factors have been suggested to be driven by ERK1/2 and Akt pathways to initiate and mediate the EMT ${ }^{11}$ and to be suppressed during MET in fibroblasts generating iPS cells..$^{9,23}$ We observed a significant $76.9 \%$ reduction in the mRNA expression of SNAI2, but not of SNAI1, ZEB1 or ZEB2 after treatment for $48 \mathrm{~h}$ with the Axl kinase inhibitor (Figure 6a). Concomitant with the reduction of mRNA expression, immunofluorescence staining confirmed that longer treatment ( 6 days) with the Axl inhibitor reduced protein expression of SNAI2 (Figure 6b). Evaluation of Axl knockdown after 3 days treatment showed a similar reduction in SNAI2 (Figures $6 \mathrm{c}$ and $\mathrm{d}$ ).

We sought to identify if other transcription factors were controlled under Axl kinase in the MET program. To do this, we first identified transcription factors that were differentially expressed between undifferentiated human lung multi-potent cell lines and freshly isolated human AEC2, a progeny of the lung multi-potent cells. Forty-one transcription factors were found to be upregulated in undifferentiated multi-potent cells compared with the AEC2 (fold change $>5, P<0.01$; Supplementary Table 3). These included SNAI2, ZEB1 and ZEB2, but not SNAI1 (Supplementary Table 3). To investigate the impact of Axl on these other transcription factors, we treated the human lung multi-potent cells with Axl inhibitor for $4 \mathrm{~h}$ and examined their gene expression levels (Figure 6e). We found that mRNA expression of six transcription factors (homeobox A5 (HOXA5), T-box 2 (TBX2), T-box 3 (TBX3), nuclear receptor subfamily 2 , group $\mathrm{F}$ member 1 (NR2F1), forkhead box F1 (FOXF1) and homeobox A3 (HOXA3)) were decreased by over $50 \%$.

\section{AxI Kinase is Relevant to Loss of Epithelial Barrier Integrity in Human Lung Diseases}

We hypothesized that if Axl-dependent signaling was a negative regulator of an alveolar epithelial cell phenotype that the Axl kinase and its ligand Gas6 should be present in human lung tissue lesions where epithelial structural integrity had been compromised. Recent human studies have suggested that such lesions are observed in epithelial hyperplasia of IPF. ${ }^{24-26}$

To address our hypothesis, we evaluated the localization of Axl in human lung alveoli where oxide and carbon dioxide are exchanged and compared this with IPF lung tissue with aberrant epithelial remodeling where such gaseous exchange is compromised. We found that in normal alveoli, without fibrosis and overt inflammation, Axl was present on endothelial cells in capillary vessels forming lumens in alveolar walls (Figure 7a). This was confirmed by colocalization of Axl and CD31 (an endothelial cell marker) (Figure 7b). However, no Axl staining was observed in the alveolar epithelial cells as these pan-cytokeratin-positive cells did not express Axl (Figure 7c). Furthermore, we confirmed the lack of expression of Axl on AEC2 identified by their cuboidal cell morphology (Figure 7a) or the expression of proSP-C (Figure 7d).

In the IPF lung tissue, HE staining showed severe destruction of alveoli and massive deposition of extracellular matrices (Figures $8 \mathrm{a}$ and $\mathrm{b}$ ). Immunohistochemistry indicated that Axl was present in epithelial hyperplasia (Figure 8c) with colocalization of the staining for Gas6 (Figure 8d). In addition, AEC2 hyperplasia, a subtype of epithelial remodeling of IPF as indicated by proSP-C staining and areas with squamous metaplasia, were positive for Axl (Figure 8e). Finally CD90-expressing epithelial cells covering fibrotic lesions in IPF, a marker of human lung multi-potent cells, ${ }^{12}$ was observed to be positive for Axl (Figure 8f).

\section{DISCUSSION}

This study has revealed an unexpected concept about the functional role of Axl, a member of the TAM (Tyro3, Axl, Mertk) receptor tyrosine kinase family. ${ }^{22}$ We demonstrate that Axl-dependent signaling has the potential to act as a negative regulator of alveolar epithelial phenotype and function. This is consistent with our observations that inhibition of Axl kinase activity, by either a small molecule inhibitor or by siRNA in human lung-derived multi-potent cells, suppresses mesenchymal cell functional properties (such as cell mobility) and promotes epithelial cell traits (such as increasing expression of epithelial adhesion molecules important in epithelial junction and surfactant proteins), which are critical for the integrity of pulmonary epithelium. We further identified a unique profile of transcription factors directly controlled under Axl kinase in the MET, which could regulate epithelial integrity in severe respiratory diseases. 
a

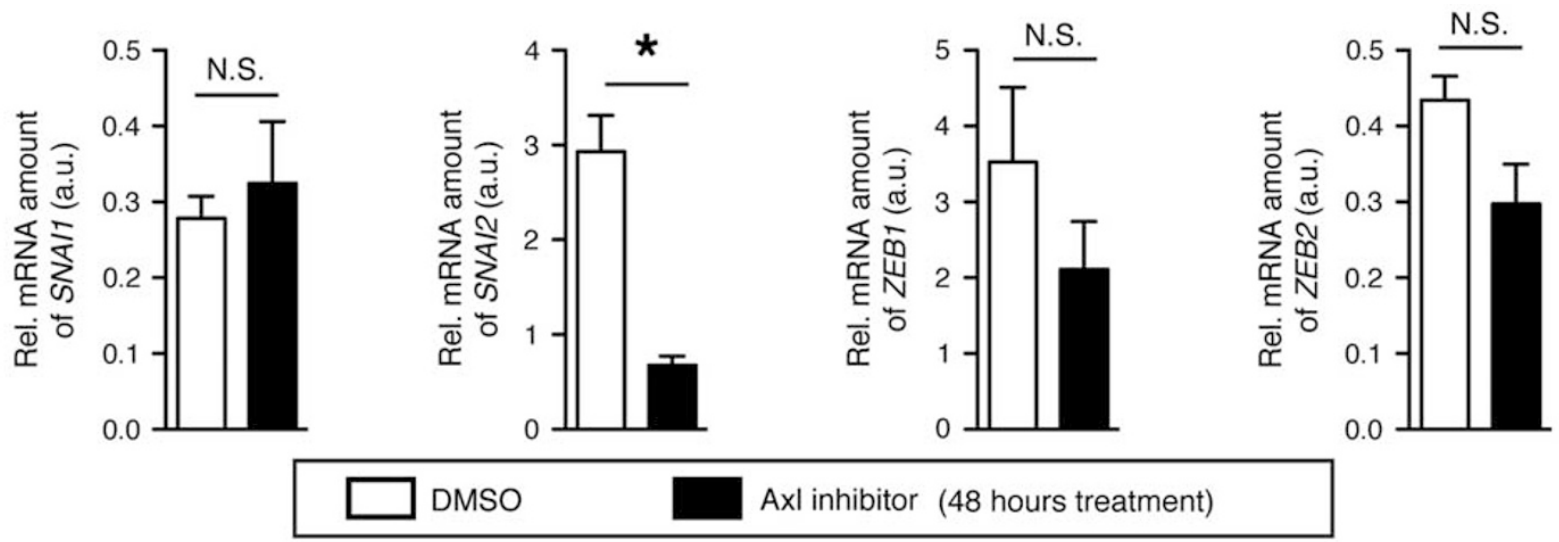

b
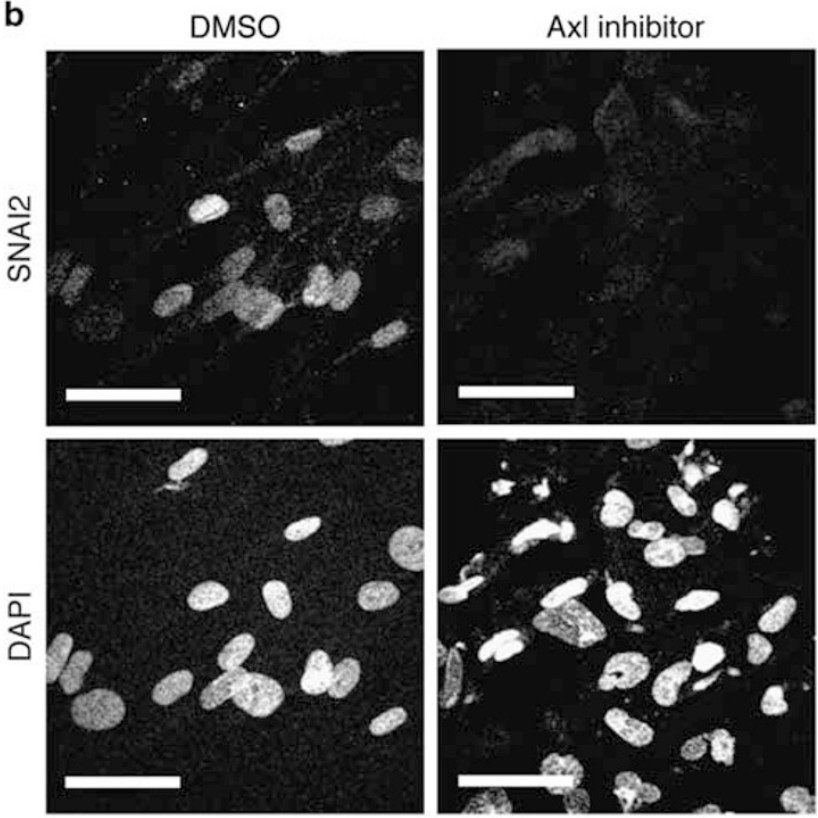

C
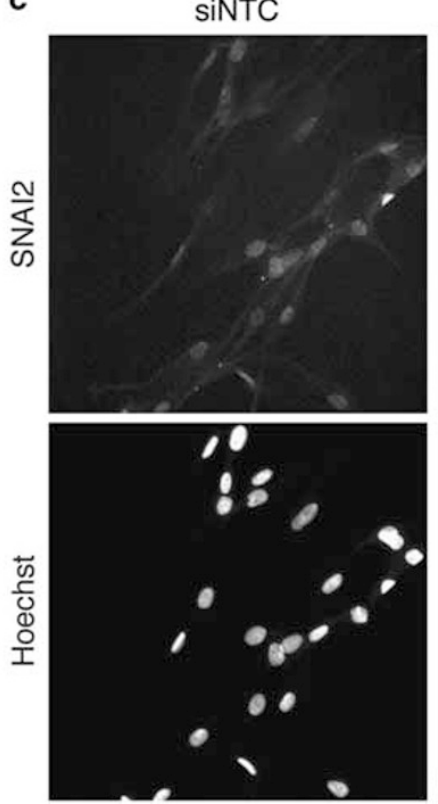

$\operatorname{siAxl}$
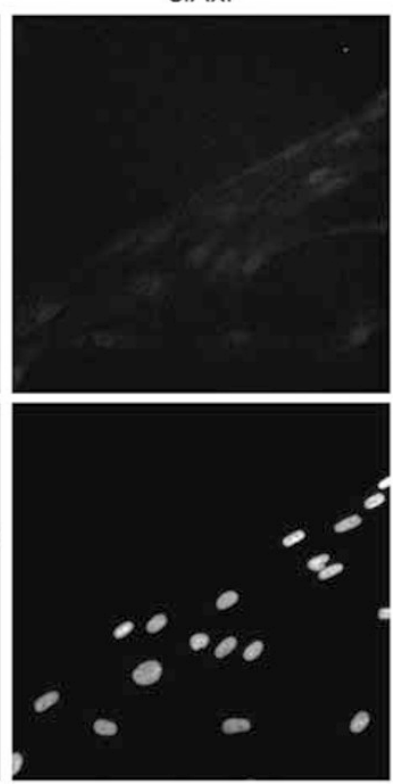

Figure 6 Involvement of EMT-mediating transcription factors in inhibition of Axl kinase. (a) qPCR analysis for transcription factors SNAl1, SNAI2, ZEB1 and ZEB2 $48 \mathrm{~h}$ after treatment of $0.1 \%$ DMSO or Axl inhibitor 2D08 $(10 \mu \mathrm{M})$. Data are mean \pm s.e.m. of three lines of human lung multi-potent cells conducted in triplicate culture. ${ }^{*} P<0.05$ between DMSO and Axl inhibitor; two-tailed unpaired $t$-test. NS means not significant. a.u. indicates arbitrary unit. (b) Representative immunostaining for SNAI2 6 days after treatment of DMSO (left panels) or Axl inhibitor 2D08 at $10 \mu \mathrm{M}$ (right panels). Nuclei were stained with DAPI. Scale bars represent $50 \mu \mathrm{m}$. (c) Representative immunostaining for SNAI2 (top) in the presence of non-targeted siRNA (siNTC) or Axl-targeted siRNA (siAxl) for three days. Nuclei were stained with Hoechst 33342 (bottom). Images were taken at $\times 20$ magnifications. (d) Image analysis showing the intensity of SNAI2 staining. Human lung multi-potent cells (Line 2) were transfected with Axl-targeted siRNAs. Untreated cells (untreat), cells treated with only lipofectamine (mock) and cells treated with non-targeted siRNA with lipofectamine (siNTC) served as transfection controls. Data are mean \pm s.d. of quadruplicate cultures. ${ }^{* *} P<0.001$ vs siNTC; one-way analysis of variance with Bonferroni's multiple comparison test. (e) $q \mathrm{PCR}$ analysis for 12 transcription factors that were identified through Affymetrix microarray as upregulated genes in human lung multi-potent cell lines compared with freshly isolated AEC2 (see Supplementary Table 3). Data are mean \pm s.d. of two independent experiments conducted in triplicate cultures of Line 1.

Our results add to the growing literature on the diverse cellular functions that Axl kinase has been associated with. These functions include: (1) driving cellular proliferation, differentiation and survival in transformed cells and nontransformed cells such as lens epithelial cells; ${ }^{27}$ (2) participating in recognition and clearance of apoptotic cells by macrophages; 22 (3) regulating innate immune response by dendritic cells, ${ }^{28}$ (4) vasculature remodeling in smooth muscle cells; ${ }^{29}(5)$ differentiation and cytokine secretion in natural killer cells; ${ }^{30}$ (6) cancer resistance through EMT program; $3^{31-35}$ and (7) mediating binding of virus to target cells. ${ }^{36}$

How Axl mediates such a diversity of roles is not completely understood but is related to how Axl is activated, its signal transduction and what other proteins it interacts with. ${ }^{37} \mathrm{Axl}$ is known to be activated by its natural ligand, Gas6, which is present within plasma ${ }^{38}$ and like other receptor tyrosine kinases (RTKs), Axl can be autophosphorylated by this Gas6-mediated dimerization. ${ }^{39}$ 

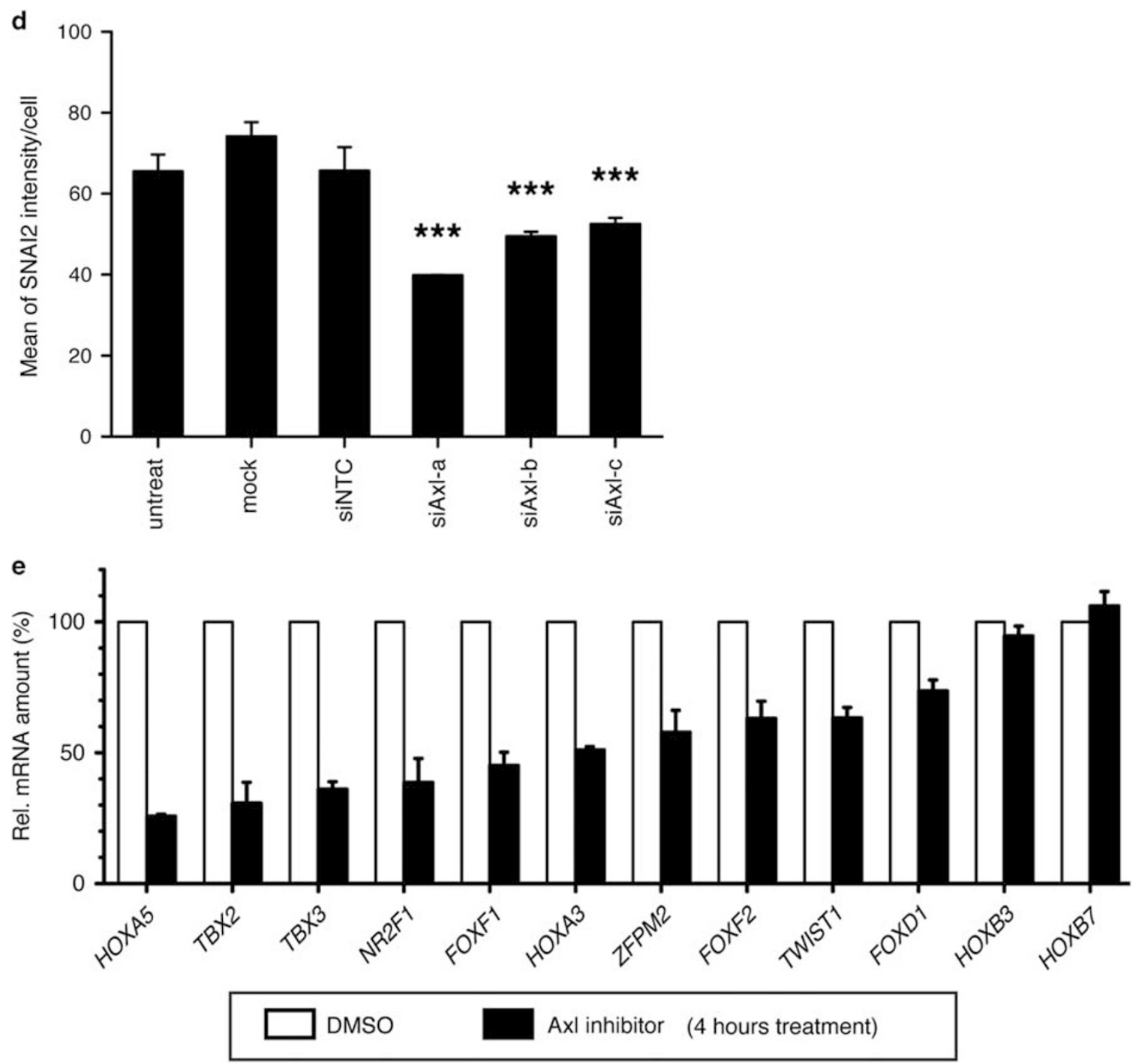

Figure 6 Continued.

However, it is reported that optimal Axl signaling also requires engagement with both Gas6 and the binding of phospholipid phosphatidylserine to the Gla domain of Gas6. ${ }^{40,41}$ In this study, Gas6 is produced by the human lung multi-potent cells and Axl phosphorylation observed can be reversed by an Axl kinase inhibitor. These results of autoactivation are consistent with previous studies using in vitro culture system of primary human pulmonary artery endothelial cells ${ }^{42}$ and human cancer cell lines. ${ }^{43,44}$ However, we cannot rule out the involvement of other RTKs such as vascular endothelial growth factor receptor (VEGFR) and epithelial growth factor receptor (EGFR), which can phosphorylate and trans-activate Axl in a Gas6-independent way, ${ }^{45,46}$ although it is less likely as VEGFR or EGFR kinase inhibitors did not alter the production of proSP-C (Supplementary Table 1).
The intracellular domain of Axl is known to interact with C1-TEN (also known as Tensin2) a focal adhesion molecule that binds to actin filaments. ${ }^{47} \mathrm{We}$ found that C1-TEN is expressed by our cells (data not shown) and through its interaction with Axl could mediate the morphological changes and the motility we see in our cells as this protein has a role in regulating cell migration. We observed that inhibition of Axl disrupted the cytoskeleton/actin filaments with a concomitant decrease in mesenchymal intermediate filaments, leading to the cells losing front-rear polarity and minimizing their ability to migrate. In addition, expression of tight junction and adherence junction proteins, as well as the translocation of $\beta$-catenin to cellular junctions were increased.

Our result identified a novel set of Axl-controlled transcription factors in the MET. This included SNAI2, HOXA5/3, TBX2/3, NR2F1 and FOXF1. Of these transcription factors, only SNAI2 has been reported to be associated 

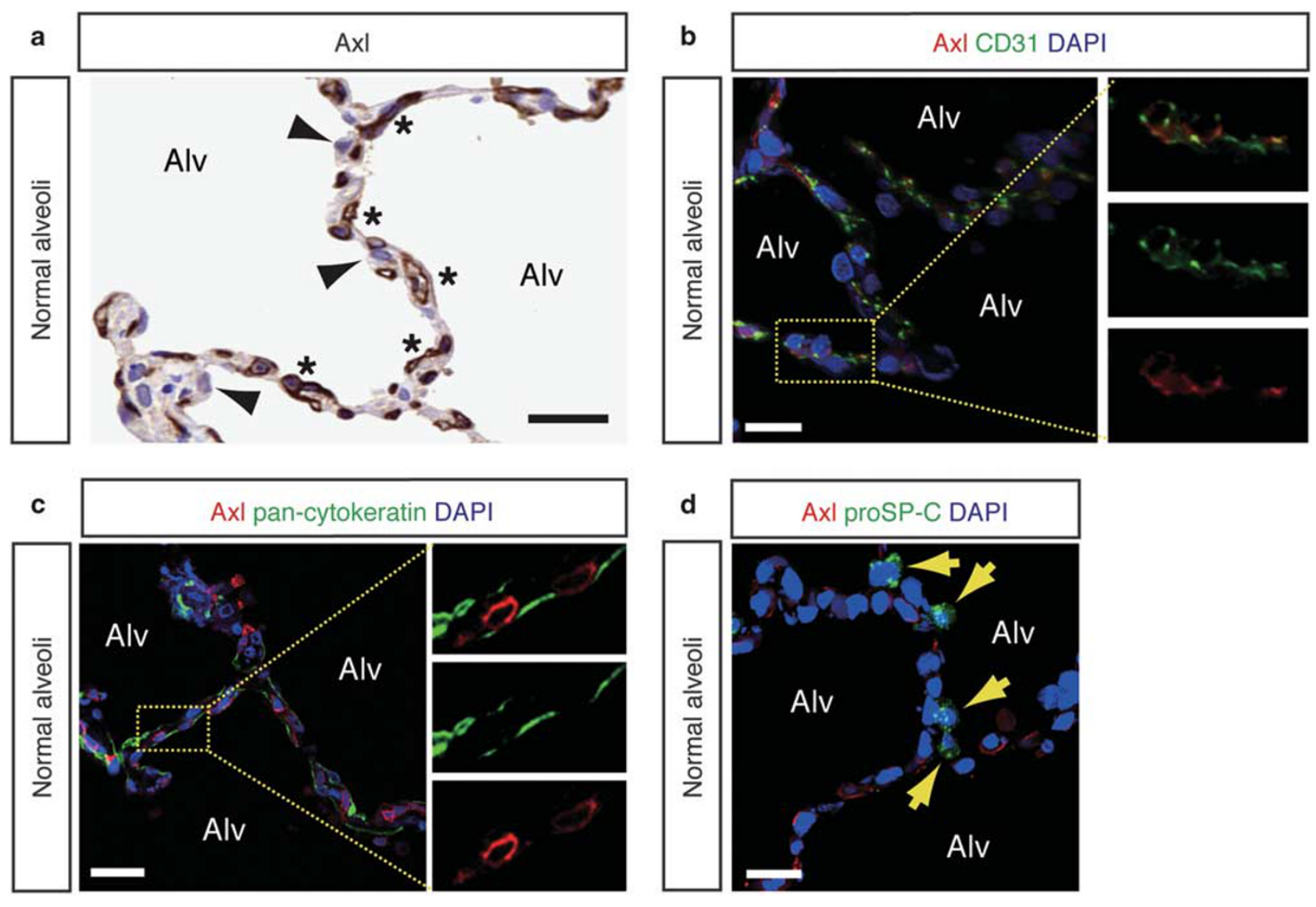

Figure 7 Localization of Axl kinase is in normal distal human lung tissues. (a) Immunohistochemistry for Axl in normal alveoli. Asterisks indicate capillary vessels forming lumens. Arrow heads show cuboidal cells located at corners of alveoli morphologically corresponding to alveolar epithelial type II cells (AEC2). Scale bar, $20 \mu \mathrm{m}$. Alv indicates an alveolar space (b) Double immunostaining for Axl (red) with an endothelial cell marker, CD31 (green) with nuclear staining by DAPI (blue). Scale bar, $20 \mu \mathrm{m}$. (c) Double immunostaining for AxI (red) with a pan epithelial cell marker, pan-cytokeratin (green) with nuclear staining by DAPI (blue). Scale bar, $20 \mu \mathrm{m}$. (d) Double immunostaining for AxI (red) with an AEC2 marker, proSP-C (green) with nuclear staining by DAPI (blue). Yellow arrows indicate AEC2. Scale bar, $20 \mu \mathrm{m}$.

with both the EMT/MET ${ }^{11}$ and Axl. ${ }^{31,48}$ HOXA5, ${ }^{49}$ TBX2, ${ }^{50}$ $\mathrm{TBX}^{51}$ and FOXF1 ${ }^{52}$ are suggested to be involved in the EMT but, to our knowledge, no report describing association of these transcription factors with Axl kinase has been established. Furthermore, we newly observe that NR2F1 and HOXA3 are Axl-controlled transcription factors, which currently are not known to be engaged in the EMT.

We observed that Axl kinase was activated in alveolar epithelial cells in areas of human IPF lung undergoing tissue fibrosis. IPF is a progressive disease of unknown cause, which is characterized as massive deposition of extracellular matrices in the lung. ${ }^{53}$ With growing insights into pathogenesis of IPF, it has been proposed that endoplasmic reticulum stress could contribute to fibrotic remodeling in the lungs, at least partly via TGF- $\beta$ activation and EMT-mediated pathways. ${ }^{8}$ Interestingly, the TGF- $\beta$ pathway has been shown to upregulate Axl expression. ${ }^{54}$ Activation of EMT in AECs alters structural and functional integrity of epithelium, ${ }^{4,5}$ drives local fibroblasts toward myofibroblasts ${ }^{55}$ or converts the AECs to myofibroblasts. ${ }^{4,56,57}$ Our in vitro and immunohistological observations suggest that in the lung Axl kinase may be involved in stabilizing mesenchymal phenotype of the epithelial cells.

How Gas6-Axl pathway is activated in human lung diseases is not yet established. Plasma levels of Gas6 were significantly elevated in adult clinical asthma of all severities compared with non-asthmatics. ${ }^{58}$ Whether these elevated levels are a reflection of lung tissue is not known. However, breakdown of the epithelial barrier could lead to increase Gas6 in the lung interstitial space with a subsequent negative effect on epithelial integrity. Alternatively reactive oxygen species (ROS) have been shown to activate Axl within a rapid time frame. ${ }^{59,60}$ ROS can be produced by epithelial cells, generated by tobacco smoke and has been associated with respiratory diseases. ${ }^{61}$ Finally, apoptotic cells, which are abundant in severe lung disease, may trigger activation of Axl. ${ }^{40,41}$ Our observations of the association of the Gas6-Axl pathway in abnormal tissue remodeling in human respiratory disease are compatible with studies of the Gas6-Axl pathway in animal models of airway remodeling. Here a murine fungal allergic 

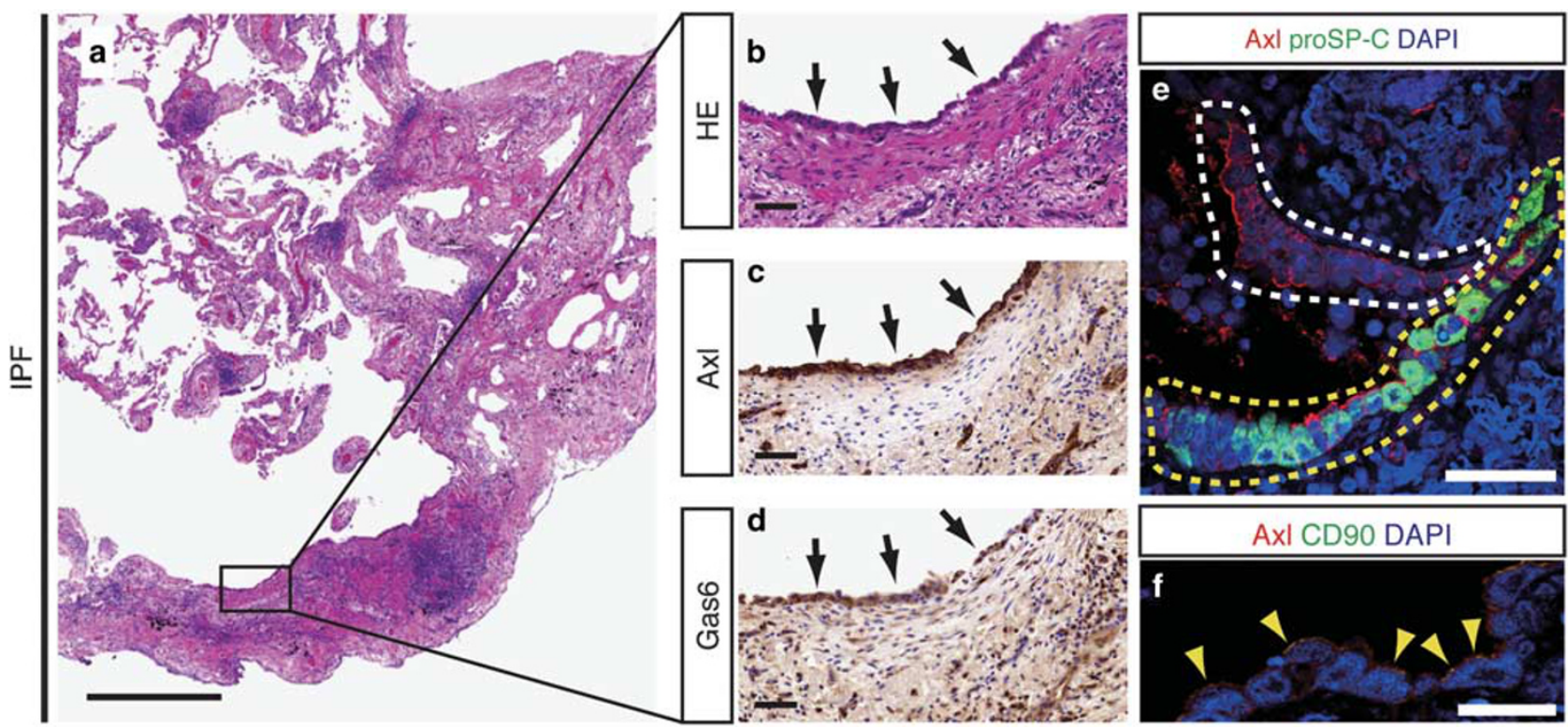

Figure $8 \mathrm{Axl}$ and Gas6 expression in epithelial remodeling in IPF. (a) Histology of a lung tissue of a patient with IPF stained for hematoxylin-eosin (HE). Scale bar, $1000 \mu \mathrm{m}$. (b-d) Sequential sections with higher magnification of the rectangle in (a) indicating HE staining (b), and immunohistochemistry for $\mathrm{Axl}$ (c) and Gas6 (d). Arrows indicate hyperplastic epithelial cells surrounding fibrotic lesions. Scale bar, $50 \mu \mathrm{m}$. (e) Double immunostaining of Axl (red) and proSP-C (green). A dashed line in yellow indicates hyperplasia of alveolar epithelial type II cells (AEC2), whereas a dashed line in white shows squamous metaplasia. (f) Double immunostaining of AxI (red) and CD90 (green) in hyperplastic AEC2. Arrow heads in yellow indicate Axl/CD90 doublepositive cells.

airway model airway remodeling was exacerbated by intranasal administration of Gas6 and ameliorated in a Gas6 knockout mouse compared with wild-type mice. ${ }^{58}$ Our results would predict that these results could be mediated by the alteration in the epithelial cell phenotype.

In summary, we found that inhibition of Axl kinase could form epithelial adhesion structure and augment surfactant protein production via the MET of human lung multi-potent cells. This was coincidental with a decrease in mesenchymal phenotypes and EMT-mediating transcription factors. Moreover, we found Axl kinase was activated in epithelial remodeling of human lung fibrotic disorder, which may lead to loss of the epithelial integrity in this severe lung diseases. Further work is required to understand whether the Axl pathway may be targeted to restore lung function.

Supplementary Information accompanies the paper on the Laboratory Investigation website (http://www.laboratoryinvestigation.org)

\section{ACKNOWLEDGMENTS}

We are especially grateful to Eylem Gürcan for excellent technical advices. We are also grateful to Johan Meuller, Ola Engkivst and Ryan Hicks for supporting the screening; to Britt-Marie Kihlberg and Alan Sabirsh for image analysis; to Amy McDonough and Lena Borjensson for compound logistics; to Lisa Öberg for microarray analysis; to Chiharu Ota for cell isolation and culture; to Takaya Suzuki and Satoshi Suzuki for obtaining human lung tissues; to Tracy Hussell and Toshifumi Fujimori for helpful discussions. This work was supported by a grant from the Japan Society for the Promotion of Science (no. 25293190) to HK. Illustration of the kinome reproduced courtesy of Cell Signaling Technology, Inc (www.cellsignal.com).

\section{DISCLOSURE/CONFLICT OF INTEREST}

NF was a post-doctoral fellow at AstraZeneca when the work was undertaken. RAM is an employee and shareholder of AstraZeneca. HK declares no conflict of interest.

1. Whitsett JA, Alenghat T. Respiratory epithelial cells orchestrate pulmonary innate immunity. Nat Immunol 2015;16:27-35.

2. Nelson WJ. Remodeling epithelial cell organization: transitions between front-rear and apical-basal polarity. Cold Spring Harb Perspect Biol 2009;1:a000513.

3. Huang RY, Guilford P, Thiery JP. Early events in cell adhesion and polarity during epithelial-mesenchymal transition. J Cell Sci 2012;125 (Pt 19):4417-4422.

4. Miyoshi K, Yanagi S, Kawahara K, et al. Epithelial Pten controls acute lung injury and fibrosis by regulating alveolar epithelial cell integrity. Am J Respir Crit Care Med 2013;187:262-275.

5. Tsujino K, Takeda Y, Arai T, et al. Tetraspanin CD151 protects against pulmonary fibrosis by maintaining epithelial integrity. Am J Respir Crit Care Med 2012;186:170-180.

6. Selman M, Pardo A. Revealing the pathogenic and aging-related mechanisms of the enigmatic idiopathic pulmonary fibrosis. an integral model. Am J Respir Crit Care Med 2014;189:1161-1172.

7. Bartis D, Mise N, Mahida RY, et al. Epithelial-mesenchymal transition in lung development and disease: does it exist and is it important? Thorax 2014;69:760-765.

8. Wolters PJ, Collard HR, Jones KD. Pathogenesis of idiopathic pulmonary fibrosis. Annu Rev Pathol 2014;9:157-179.

9. Samavarchi-Tehrani P, Golipour A, David L, et al. Functional genomics reveals a BMP-driven mesenchymal-to-epithelial transition in the initiation of somatic cell reprogramming. Cell Stem Cell 2010;7:64-77.

10. Sakurai K, Talukdar I, Patil VS, et al. Kinome-wide functional analysis highlights the role of cytoskeletal remodeling in somatic cell reprogramming. Cell Stem Cell 2014;14:523-534.

11. Lamouille S, Xu J, Derynck R. Molecular mechanisms of epithelialmesenchymal transition. Nat Rev Mol Cell Biol 2014;15:178-196.

12. Fujino N, Kubo H, Suzuki T, et al. Isolation of alveolar epithelial type II progenitor cells from adult human lungs. Lab Invest 2011;91:363-378. 
13. Phelps DS, Floros J. Localization of pulmonary surfactant proteins using immunohistochemistry and tissue in situ hybridization. Exp Lung Res 1991;17:985-995.

14. Beers MF, Kim CY, Dodia C, et al. Localization, synthesis, and processing of surfactant protein SP-C in rat lung analyzed by epitope-specific antipeptide antibodies. J Biol Chem 1994;269:20318-20328.

15. Hoing S, Rudhard $Y$, Reinhardt $P$, et al. Discovery of inhibitors of microglial neurotoxicity acting through multiple mechanisms using a stem-cell-based phenotypic assay. Cell Stem Cell 2012;11:620-632.

16. Chartier M, Chenard T, Barker J, et al. Kinome Render: a stand-alone and web-accessible tool to annotate the human protein kinome tree. PeerJ 2013;1:e126.

17. Ghaedi M, Calle EA, Mendez JJ, et al. Human iPS cell-derived alveolar epithelium repopulates lung extracellular matrix. J Clin Invest 2013;123:4950-4962.

18. Huang SX, Islam MN, O'Neill J, et al. Efficient generation of lung and airway epithelial cells from human pluripotent stem cells. Nat Biotechnol 2014;32:84-91.

19. Desai TJ, Brownfield DG, Krasnow MA. Alveolar progenitor and stem cells in lung development, renewal and cancer. Nature 2014;507: 190-194.

20. O'Bryan JP, Frye RA, Cogswell PC, et al. axl, a transforming gene isolated from primary human myeloid leukemia cells, encodes a novel receptor tyrosine kinase. Mol Cell Biol 1991;11:5016-5031.

21. Thiery JP, Sleeman JP. Complex networks orchestrate epithelialmesenchymal transitions. Nat Rev Mol Cell Biol 2006;7:131-142.

22. Lemke G. Biology of the TAM receptors. Cold Spring Harb Perspect Biol 2013;5:a009076.

23. Li R, Liang J, Ni S, et al. A mesenchymal-to-epithelial transition initiates and is required for the nuclear reprogramming of mouse fibroblasts. Cell Stem Cell 2010;7:51-63.

24. Kim KK, Kugler MC, Wolters PJ, et al. Alveolar epithelial cell mesenchymal transition develops in vivo during pulmonary fibrosis and is regulated by the extracellular matrix. Proc Natl Acad Sci USA 2006;103:13180-13185.

25. Marmai C, Sutherland RE, Kim KK, et al. Alveolar epithelial cells express mesenchymal proteins in patients with idiopathic pulmonary fibrosis. Am J Physiol Lung Cell Mol Physiol 2011;301:L71-L78.

26. Willis BC, Liebler JM, Luby-Phelps $\mathrm{K}$, et al. Induction of epithelialmesenchymal transition in alveolar epithelial cells by transforming growth factor-beta1: potential role in idiopathic pulmonary fibrosis. Am J Pathol 2005;166:1321-1332.

27. Collett G, Wood A, Alexander MY, et al. Receptor tyrosine kinase Axl modulates the osteogenic differentiation of pericytes. Circ Res 2003;92: 1123-1129.

28. Rothlin CV, Ghosh S, Zuniga El, et al. TAM receptors are pleiotropic inhibitors of the innate immune response. Cell 2007;131:1124-1136.

29. Korshunov VA. Axl-dependent signalling: a clinical update. Clin Sci (Lond) 2012;122:361-368.

30. Park IK, Giovenzana $C$, Hughes $T L$, et al. The Axl/Gas6 pathway is required for optimal cytokine signaling during human natural killer cell development. Blood 2009;113:2470-2477.

31. Asiedu MK, Beauchamp-Perez FD, Ingle JN, et al. AXL induces epithelial-to-mesenchymal transition and regulates the function of breast cancer stem cells. Oncogene 2014;33:1316-1324.

32. Cichon MA, Szentpetery Z, Caley MP, et al. The receptor tyrosine kinase Axl regulates cell-cell adhesion and stemness in cutaneous squamous cell carcinoma. Oncogene 2014;33:4185-4192.

33. Leconet W, Chentouf M, Du Manoir S, et al. Therapeutic activity of antiAXL antibody against triple-negative breast cancer patient derived xenografts and metastasis. Clin Cancer Res 2016; doi: 10.1158/10780432.CCR-16-1316.

34. Zhang $Z$, Lee JC, Lin $L$, et al. Activation of the AXL kinase causes resistance to EGFR-targeted therapy in lung cancer. Nat Genet 2012;44: 852-860.

35. Yen SY, Chen SR, Hsieh J, et al. Biodegradable interstitial release polymer loading a novel small molecule targeting Axl receptor tyrosine kinase and reducing brain tumour migration and invasion. Oncogene 2016;35:2156-2165.

36. Morizono $\mathrm{K}, \mathrm{Xie} \mathrm{Y}$, Olafsen $\mathrm{T}$, et al. The soluble serum protein Gas6 bridges virion envelope phosphatidylserine to the TAM receptor tyrosine kinase Axl to mediate viral entry. Cell Host Microbe 2011;9: 286-298.
37. Graham DK, DeRyckere D, Davies KD, et al. The TAM family: phosphatidylserine sensing receptor tyrosine kinases gone awry in cancer. Nat Rev Cancer 2014;14:769-785.

38. Balogh I, Hafizi S, Stenhoff J, et al. Analysis of Gas6 in human platelets and plasma. Arterioscler Thromb Vasc Biol 2005;25:1280-1286.

39. Sasaki T, Knyazev PG, Clout NJ, et al. Structural basis for Gas6-Ax signalling. EMBO J 2006;25:80-87.

40. Lew ED, Oh J, Burrola PG, et al. Differential TAM receptor-ligandphospholipid interactions delimit differential TAM bioactivities. Elife 2014;3:e03385.

41. Zagorska A, Traves PG, Lew ED, et al. Diversification of TAM receptor tyrosine kinase function. Nat Immunol 2014;15:920-928.

42. Healy AM, Schwartz JJ, Zhu X, et al. Gas 6 promotes Axl-mediated survival in pulmonary endothelial cells. Am J Physiol Lung Cell Mol Physiol 2001;280:L1273-L1281.

43. Avilla $\mathrm{E}$, Guarino V, Visciano $\mathrm{C}$, et al. Activation of TYRO3/AXL tyrosine kinase receptors in thyroid cancer. Cancer Res 2011;71:1792-1804.

44. Li Y, Ye X, Tan C, et al. Axl as a potential therapeutic target in cancer: role of Axl in tumor growth, metastasis and angiogenesis. Oncogene 2009;28:3442-3455.

45. Meyer AS, Miller MA, Gertler FB, et al. The receptor AXL diversifies EGFR signaling and limits the response to EGFR-targeted inhibitors in triplenegative breast cancer cells. Sci Signal 2013;6:ra66.

46. Ruan GX, Kazlauskas A. Axl is essential for VEGF-A-dependent activation of PI3K/Akt. EMBO J 2012;31:1692-1703.

47. Hafizi $S$, Alindri $F$, Karlsson $\mathrm{R}$, et al. Interaction of Axl receptor tyrosine kinase with C1-TEN, a novel C1 domain-containing protein with homology to tensin. Biochem Biophys Res Commun 2002;299: 793-800.

48. Koorstra JB, Karikari CA, Feldmann G, et al. The Axl receptor tyrosine kinase confers an adverse prognostic influence in pancreatic cancer and represents a new therapeutic target. Cancer Biol Ther 2009;8: 618-626.

49. Cieply B, Farris J, Denvir J, et al. Epithelial-mesenchymal transition and tumor suppression are controlled by a reciprocal feedback loop between ZEB1 and Grainyhead-like-2. Cancer Res 2013;73:6299-6309.

50. Wang $B$, Lindley LE, Fernandez-Vega $V$, et al. The $T$ box transcription factor TBX2 promotes epithelial-mesenchymal transition and invasion of normal and malignant breast epithelial cells. PLoS ONE 2012;7: e41355.

51. Rodriguez $\mathrm{M}$, Aladowicz $\mathrm{E}$, Lanfrancone $\mathrm{L}$, et al. Tbx3 represses E-cadherin expression and enhances melanoma invasiveness. Cancer Res 2008;68:7872-7881.

52. Nilsson J, Helou K, Kovacs A, et al. Nuclear Janus-activated kinase 2/ nuclear factor 1-C2 suppresses tumorigenesis and epithelial-tomesenchymal transition by repressing Forkhead box F1. Cancer Res 2010;70:2020-2029.

53. King Jr. TE, Pardo A, Selman M. Idiopathic pulmonary fibrosis. Lancet 2011;378:1949-1961.

54. Bauer T, Zagorska A, Jurkin J, et al. Identification of Axl as a downstream effector of TGF-beta1 during Langerhans cell differentiation and epidermal homeostasis. J Exp Med 2012;209:2033-2047.

55. Yang J, Wheeler SE, Velikoff $M$, et al. Activated alveolar epithelial cells initiate fibrosis through secretion of mesenchymal proteins. Am J Pathol 2013;183:1559-1570.

56. Kim KK, Wei Y, Szekeres C, et al. Epithelial cell alpha3beta1 integrin links beta-catenin and Smad signaling to promote myofibroblast formation and pulmonary fibrosis. J Clin Invest 2009;119:213-224.

57. Balli D, Ustiyan $V$, Zhang $Y$, et al. Foxm 1 transcription factor is required for lung fibrosis and epithelial-to-mesenchymal transition. EMBO J 2013:32:231-244.

58. Shibata T, Ismailoglu UB, Kittan NA, et al. Role of growth arrest-specific gene 6 in the development of fungal allergic airway disease in mice. Am J Respir Cell Mol Biol 2014;51:615-625.

59. Konishi A, Aizawa T, Mohan A, et al. Hydrogen peroxide activates the Gas6-Axl pathway in vascular smooth muscle cells. J Biol Chem 2004;279:28766-28770.

60. Valverde P. Effects of Gas6 and hydrogen peroxide in Axl ubiquitination and downregulation. Biochem Biophys Res Commun 2005:333:180-185.

61. Gao W, Li L, Wang Y, et al. Bronchial epithelial cells: the key effector cells in the pathogenesis of chronic obstructive pulmonary disease? Respirology 2015;20:722-729. 